\title{
Effective medium theory for anisotropic media with plasmonic core-shell nanoparticle inclusions
}

\author{
V.Yu. Reshetnyak ${ }^{1}$, I.P. Pinkevych ${ }^{1}$, T.J. Sluckin ${ }^{2}$, a A.M. Urbas ${ }^{3}$, and D.R. Evans ${ }^{3}$ \\ 1 Physics Faculty, National Taras Shevchenko University of Kyiv, 64 Volodymyrska Street, Kyiv 01601, Ukraine \\ 2 School of Mathematical Sciences, University of Southampton, Southampton, SO17 1BJ, UK \\ 3 Air Force Research Laboratory, Materials and Manufacturing Directorate, Wright-Patterson Air Force Base, Ohio 45433, USA
}

Received: 22 August 2018

Published online: 19 September 2018

(c) The Author(s) 2018. This article is published with open access at Springerlink.com

\begin{abstract}
Core-shell building blocks have been found useful in recent years as inclusions, in the search for metamaterials with tailored properties. Either the core or the shell of these composite inclusions may be metallic, and the dielectric component may be both radially anisotropic and radially inhomogeneous. In tunable anisotropic metamaterials, the tuning may then be achieved through the host, the core, or some combination thereof. However a theoretical picture is harder to build. Here we propose an approach to an effective medium theory for such materials, valid in the quasi-static limit. The method proceeds first by homogenising the interior of complex particle, and then uses standard anisotropic effective medium methods to provide bulk effective homogenized parameters. By varying the degree of inhomogeneity in the core, shell and dielectric-metal material volume fractions, the technique can be used as a tool for the design of metamaterials with specifically engineered properties. We find that metamaterial properties can be readily tuned by reorienting the optical axis of the host (e.g., liquid crystal). In particular, there is a possibility of switching between hyperbolic and conventional anisotropic metamaterial properties by changing inclusion shell properties.
\end{abstract}

\section{Introduction}

Over recent years it has been increasingly the case that natural materials do not possess properties sufficiently versatile to satisfy modern demanding engineering requirements. Composites, typically containing colloidal inclusions inside a host material whose properties are known, enable artificial materials to combine some properties, and eliminate others, possessed by the constituent components $[1,2]$. By this means novel materials with specific sets of required properties can be fabricated. In particular, in an optical and electronic context, these systems are often labelled metamaterials, and as such attract intense interest in the physics, optics, and engineering communities [3-5].

The range of composite material properties can be further enriched and tuned if the inclusions themselves consist of composite particles. Such particles typically contain a core and a shell with opposing tendencies. A non-exhaustive list includes: a gold core surrounded by a silica shell [6], a silica core covered with a gold semi-shell [7], metallic cores inside a semiconductor shell [8], and indeed Janus particles in general, which consist of particles with patches with distinct chemical properties [9]. Bulk properties can now be tuned by changing the properties of the host, the concentration of inclusions, and of course, the detailed internal structure of complex nanoparticle inclusions. One dramatic example occurs when either the core or shell is built from a material going through a metal-insulator phase transition, such as germanium antimony telluride [10], or vanadium dioxide [11]. The space of potential properties is vast, and engineering design also requires reliable tools for material property prediction. However, the challenge involved in calculating bulk properties of complex metamaterials is significant, particularly when, as in the cases considered in this paper, the host materials are also anisotropic.

In this paper we provide a simple route to describe the effective dielectric properties of anisotropic media composed of an anisotropic host with core-shell inclusions, in which the shell or core may itself be both inhomogeneous and anisotropic. Our work differs from previous work both in the anisotropy of the host and the anisotropic properties of the inclusions. The inclusions themselves can be either spherical or cylindrical. Complex inclusion shape, intrinsic

\footnotetext{
a e-mail: t.j.sluckin@soton.ac.uk
} 
anisotropy and internal inclusion structure are all elements which complicate calculations. Calculations involving all three present an extra burden which can only be overcome at the moment by numerical methods.

We shall suppose the internal anisotropy to be radially inhomogeneous with principal axis in the radial direction. This is a simplifying hypothesis, but in fact there are also experimental systems which appear to possess this property. For example, Erbe and Sigel [12] have discovered the anisotropy in unilamellar phospholipid vesicles; de Beule [13] has addressed experimentally surface scattering of core-shell particles with anisotropic shells, while Golovin and Lavrentovich [14] observed a spatially varying refractive index in a system of Au nanorods placed in an inhomogeneous electric field with radial symmetry. In this latter case, the rods aligned along the electric field create an optically birefringent cloud near the cylindrical electrode.

Anisotropic metamaterials present an extra theoretical challenge. in that the theory required to describe the bulk properties of a composite with anisotropic inclusions is less well-founded. Anisotropy presents extra calculational complexity, but at the same time, provides extra degrees of freedom which can be used to control material properties. The potential advantages of all such materials make them extremely attractive for applications, in particular if the host is liquid crystalline and the inclusions are themselves anisotropic.

In such cases the metamaterial properties themselves may in addition be tunable in situ. However, the use of liquids in composite materials also presents some disadvantages, in that liquids need to be sealed and oriented. We hypothesize that these disadvantages could be overcome by the use of liquid crystal elastomers, which combine liquid crystalline orientational properties with rubber-like solid properties, and can be controlled with ultraviolet or visible light, by thermofields, as well as by elastic and acoustic means [15]. A particular example which has received significant attention recently occurs in uniaxial materials for which the product of the principal dielectric components is negative - these are the so-called hyperbolic metamaterials [16]. These materials have, for instance, the potential to be employed in devices which break the diffraction limit [17].

The theoretical task involves an input of the dielectric tensors at the relevant frequencies of the host, and all components of the inclusions, together with the component volume fractions and geometrical structure of the inclusions. The output is an effective dielectric tensor (which may, of course, include an imaginary part) for the medium as a whole [1824]. The procedure replaces the detailed optics of an inhomogeneous medium with known statistical properties by that of an effective homogeneous but anisotropic medium. Given the inputs, it is possible to solve Maxwell's equations. To obtain physically meaningful results, it may also be necessary to average over a suitable ensemble of equivalent systems. Scattering by inclusions involves Mie scattering or generalizations thereof [25]. Full solutions are difficult for a single particle, and impractically computationally intensive if repeated for an ensemble of a large number of particles. In practice approximations are necessary. For large particles and short light wavelengths, ray-tracing can be fruitful.

However, we are treating the opposite limit, in which light wavelengths are long compared with particle sizes and distances between particles. In this limit, the earliest attempts at homogenization, going back to the early years of the 20th century, due to Garnett [26] in 1904 and Bruggeman [27] in 1935, have by now become classics. Although details of different versions of these theories differ, the effect is to construct effective host medium parameters in such a way that an average inclusion is in some sense mathematically invisible in the effective medium.

Early versions of effective medium theory were essentially heuristic. However, more recently considerable effort has been exerted to provide a sound theoretical foundation (see, e.g., [28-30]). Specifically, Bergman [31] showed that all effective medium theories could be regarded in some sense as equivalent, but involving different approximations of a spectral function $g(x)$ which describes the microgeometry (see also [2], p41ff). Although the foundation is more solid in the case of isotropic inclusions in isotropic media, much recent work has also concentrated on systems in which the inclusions are non-spherical, or when the properties of the host medium and/or the inclusion itself possess anisotropic dielectric properties. In the cases of which we are aware, when anisotropy is included, the inclusion is structureless and uniform. Good recent reviews can be found in $[1,2,32-34]$.

The enormous attention given to core-shell systems in recent years is in part the result of the dramatic plasmonic response of a metallic core. The optical response of the whole core-shell system in a host medium is itself a non-trivial property to calculate. The procedure has been well-reviewed in the case of gold nanoparticles by Myroshnychenko et al. [35]. The dielectric properties of the material as a whole are mainly governed by those of the host properties, but with a strongly frequency-dependent structure close to the core plasmonic resonance. It is this feature which underlies the emergent metamaterial properties.

Many authors have used effective medium theories to predict metamaterial properties $[3,5,21]$. In some case, these studies use effective medium theories, but for any analytic tractability to be achieved, the inclusion properties must be isotropic. Other workers have studied light scattering from complex structures, but often these studies are constrained to be numerical, precisely because of the complexity of the problem, and it is difficult to replicate this work without access to the code and commercial software packages on which the studies are carried out. Yet other workers study specific (sometimes quite large) nanostructures using explicit optical solvers [36]. They then derive material properties by gluing together periodic copies of scatterers embedded in a host (see, e.g., [37]). In this case, at least in the view of the present authors, the benefit of solving some optics exactly may be counterbalanced by inexact averaging of randomly placed inclusions. An alternative simulation strategy consists of considering the dielectric properties of a whole collection of 
particles, then averaging over particle positions, weighting positional correlations correctly according to, say, a Gibbs ensemble, and finally looking at increasingly large systems in order to carry out a finite-size scaling analysis to determine the macroscopic limit. Although some elements of this approach have been tried for simple scatterers in two dimensions (see, e.g., [38]), a convincing implementation for complex scatterers seems unattainable in three dimensions with current computational resources. A further point concerns the difficulty of treating exactly dielectric properties of a confined electronic system. Thus, for example, some authors use a Drude model to treat the electrons in the core [37]. This neglects entirely the experimentally verified effect of localization on electron mean path and plasmonic resonance [39].

We note that the study of metamaterial properties in general is closely related to that of optical cloaking [40], in which the optical signature of particles inserted in a medium may be physically greatly reduced, or indeed in favourable circumstances made to disappear entirely, if the dielectric properties of ensembles of which they are a part are suitably tuned. Cloaking ideas are not restricted to optics, but can be extended to acoustics [41,42] and even thermodynamics [43].

Previous work has either treated anisotropy of the host and the inclusion or treated core-shell effects, but not both together. By comparison with usual articulations of these theories, the problem that we discuss contains extra complexity. This complexity is a result firstly of the need to include correctly size-dependent core parameters, secondly of the inhomogeneity of the particulate inclusions, and thirdly of the optical anisotropy associated with some or all of the components of the composite.

Thus in this paper, we combine effects which have not previously to our knowledge been studied together, specifically anisotropy in the host and the shell, electron mean-free path effects in the core, and inhomogeneity in the shell. We have in addition treated an inverted system in which the shell rather than the core is metallic. The study also includes some examples of hyperbolic switching, a phenomenon which requires the bulk dielectric properties not only to be anisotropic, but also to possess one negative component. A principal advantage of our work is that the input formulae are analytic, and relatively trivial numerical methods are required in order to apply the results to systems of interest other than those which we use in our examples. The focus of these calculations is on metamaterial properties, but we note also possible applications in biosensors based on surface plasmonic resonance [44, 45].

The organization of the paper is as follows. In sect. 2 we treat the dielectric properties of the metallic cores. We use the result of this section in sect. 3 to derive results for effective dielectric properties of structured core-shell particles embedded in a host medium. Then in sect. 4, we use the results of sect. 3 to derive an effective medium theory for ensembles of particles. In sect. 5 we use the theory in some numerical examples to show how the theory can be employed in practice. In sect. 6 we discuss the case in which the metal is in the shell rather than in the core of the embedded particle. Finally, in sect. 7 we draw some conclusions and discuss how the results can be applied.

\section{Dielectric properties of metallic cores}

In principle, input values for each component can be measured for bulk samples [46]. In the particular case of metals, there is pronounced frequency dependence. In order to be able to make practical use of standard cited bulk values (see, e.g., [47]), Etchegoin et al. [48] introduced an analytic approximation matching this dependence very closely over a broad range of wavelengths. The Etchegoin fit uses a Drude model to describe electron lifetime, a measured plasma frequency, accounts for interband transitions, but only describes bulk properties:

$$
\epsilon_{A u}(\lambda)=\epsilon_{\infty}\left(1-\frac{1}{\lambda_{p}^{2}\left(\lambda^{-2}+i\left(\mu_{p} \lambda\right)^{-1}\right)}\right)+\sum_{i=1,2} \frac{A_{i}}{\lambda_{i}}\left[\frac{e^{i \phi_{i}}}{\lambda_{i}^{-1}-\lambda^{-1}-i \mu_{p}^{-1}}+\frac{e^{-i \phi_{i}}}{\lambda_{i}^{-1}+\lambda^{-1}+i \mu_{p}^{-1}}\right],
$$

where $\lambda_{p}$ is the plasma wavelength, and $\mu_{p},\left\{A_{i}, \lambda_{i}, \mu_{i}, \phi_{i}\right\}(i=1,2)$ are fitting parameters.

For samples of finite size, the recipe must be further modified [49], primarily because scattering from the sample surface reduces the mean free path $L_{\text {eff }}$ of conduction electrons. This effect is most important when the particle dimension is reduced below the bulk mean free path. The modified formula for $\epsilon$ reads

$$
\epsilon_{l}(\omega)=\epsilon_{b}(\omega)+\frac{\omega_{p}^{2}}{\omega\left(\omega+i \gamma_{0}\right)}-\frac{\omega_{p}^{2}}{\omega(\omega+i \gamma)}
$$

Here $\omega$ is the frequency of the incident field, $\omega_{p}$ is the plasma frequency, $\epsilon_{b}$ is the experimentally determined permittivity for a bulk sample, $v_{F}$ is the mean electron velocity at the Fermi surface, $\gamma_{0}$ is the electron relaxation rate in the metal, $2 l$ is the characteristic diameter of the particle in the direction of the field, and

$$
\gamma=\gamma_{0}+\frac{A v_{F}}{L_{\mathrm{eff}}}
$$

A good estimate of the mean free path [49] is $L_{\text {eff }}=4 V / S \sim l$, where $V, S, l$ are, respectively, the volume, surface area, and dimensional scale of the sample. 


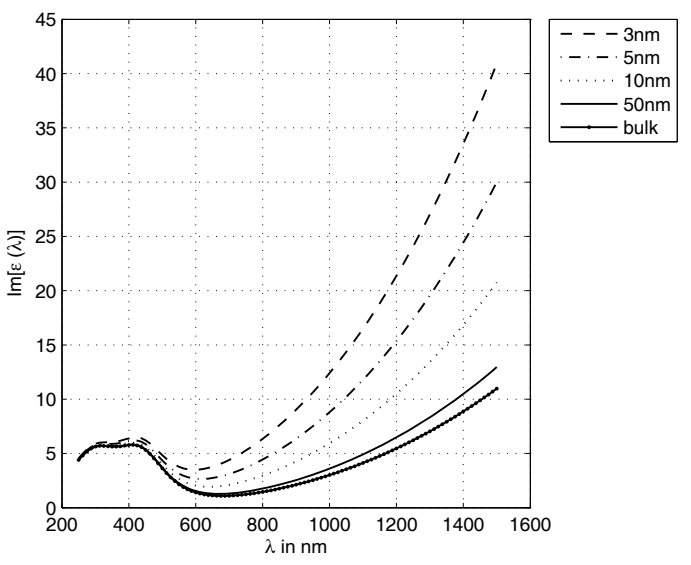

(a)

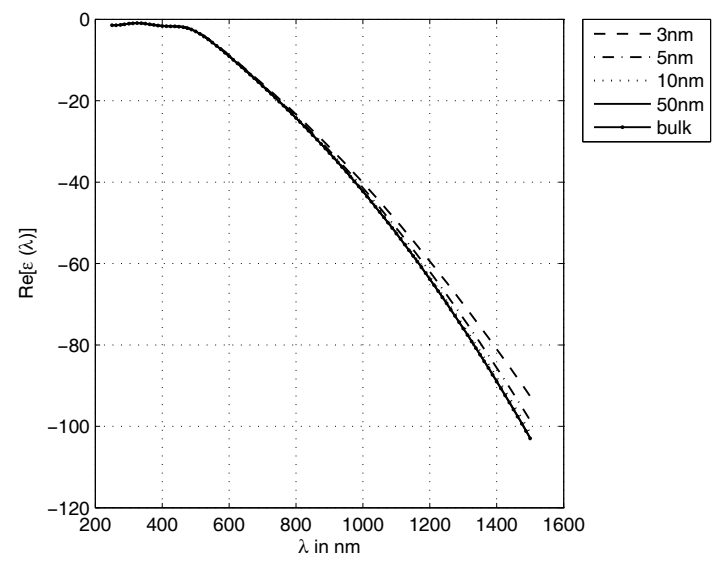

(b)

Fig. 1. Dielectric functions of spherical Au nanoparticles as a function of wavelength. (a) Imaginary part. (b) Real part. Curves correspond (as marked) to radii $3 \mathrm{~nm}, 5 \mathrm{~nm}, 10 \mathrm{~nm}, 50 \mathrm{~nm}$, and bulk gold. Mean free path from ref. [49]. Note finite-size effects on imaginary part are much more significant than on real part of dielectric permittivity.

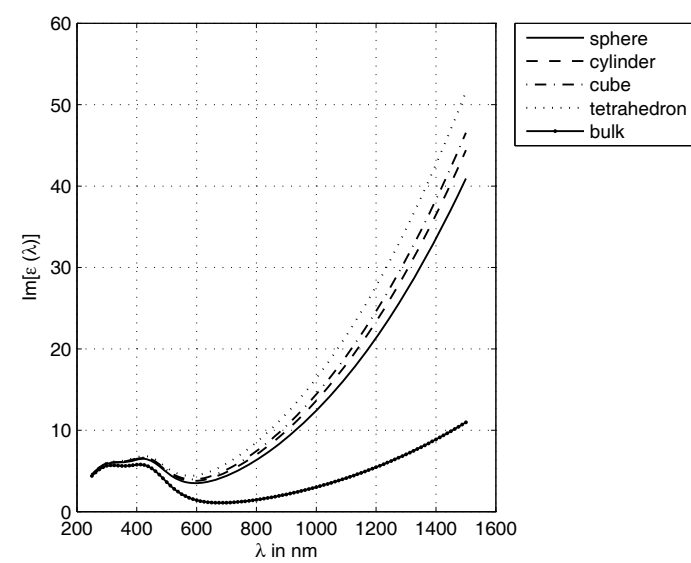

(a)

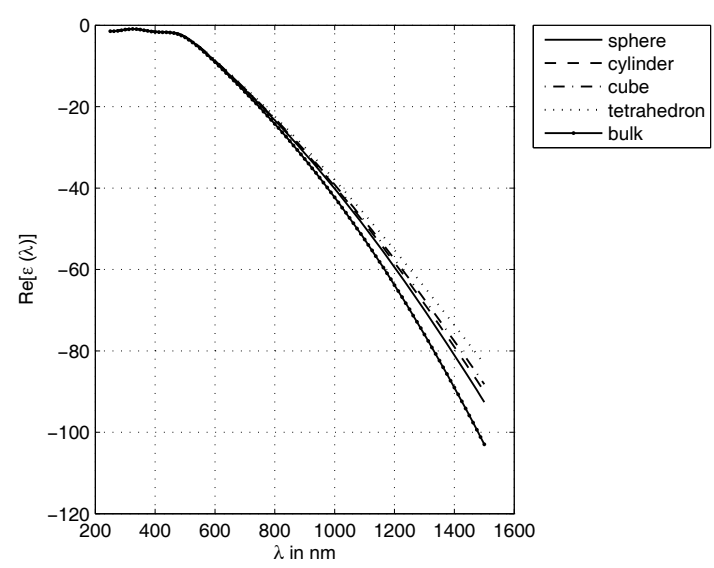

(b)

Fig. 2. Dielectric functions of Au particles with different shapes. All particles have same equivalent volume, corresponding to a sphere of diameter $6 \mathrm{~nm}$, apart from bulk system. (a) Imaginary part. (b) Real part. Dot-dash line: cube (edge length $4.84 \mathrm{~nm}$ ). Thin line: sphere. Dashed line: cylinder (height $5.24 \mathrm{~nm}$, diameter $5.24 \mathrm{~nm}$ ). Dotted line: tetrahedron (height $8.05 \mathrm{~nm}$, perpendicular bisector $8.54 \mathrm{~nm}$ ). Full line: bulk. Examples chosen so as to match examples in ref. [49], which discusses Ag particles.

Both classical and quantum methods have been used to calculate this effect [50], but in either case, the robust conclusion is that the reduction in $L_{\text {eff }}$ depends not only on sample size, but also significantly on sample shape, and even on sample topology [51]. This dependence will be important in our calculation of effective medium properties below.

As an illustrative case, we show in fig. 1 results of calculations on spherical Au nanoparticles of various radii between $3 \mathrm{~nm}$ and bulk systems. As might be expected the finite-size effects are more strongly manifested in the imaginary part, but Kramers-Kronig effects feed back into the real part as well. For the wavelengths we consider, gold nanoparticles of radius $50 \mathrm{~nm}$ have dielectric properties close to those of bulk materials. We also note that shape effects on the core dielectric constant are not negligible, as is shown in fig. 2.

\section{Single-particle properties}

In order to discuss how to treat inhomogeneous nanoparticles, we consider here a core-shell particle, with core radius $b$ and total radius $a$, in the quasi-static limit, placed for the moment in a host medium. We shall often be interested in the ratio of the volume of the core to the volume of the total core-shell particle. This is $f=(b / a)^{3}$, which in the context of complex nanoparticle colloids, is known as the filling factor. The geometry is given in fig. 3 . 


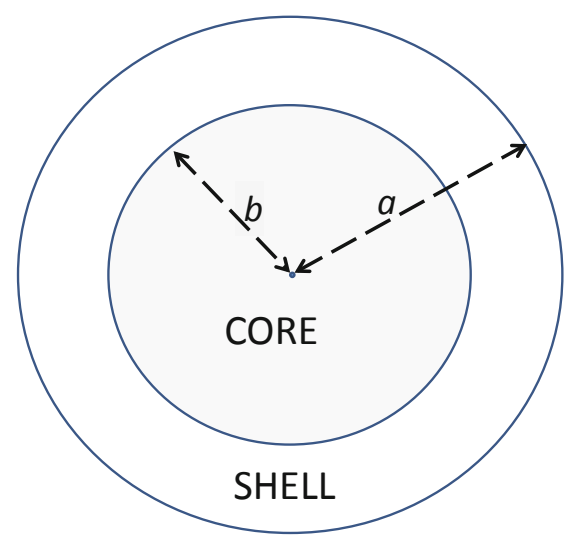

Fig. 3. Geometry of core and shell, showing radii $b$ and $a$ used in calculations.

The Au core, with dielectric constant $\epsilon_{c}$, is enclosed by a dielectric shell. The shell is radially anisotropic and inhomogeneous, where following previous work on cloaking individual particles [52], we suppose the inhomogeneity to take a specific algebraic power law form. Thus the dielectric tensor $\boldsymbol{\epsilon}(\mathbf{r})=\boldsymbol{\epsilon}(r)$ has radial and transverse principal components $\epsilon_{\|} \neq \epsilon_{\perp} ; \epsilon_{\|} r=\epsilon_{\|, 0}(r / a)^{m} ; \epsilon_{\perp}=\epsilon_{\perp, 0}(r / a)^{m}$. In principle the exponent $m$ can be either positive or negative. This form has several practical advantages. It is (a) analytically soluble, (b) a good fit to a monotonic functions in an annular shell, (c) of a similar form to that considered in a cloaking context by a number of other authors [53,54], and (d) consistent with forms used, e.g., in transformation thermodynamics [43] and optics [54] contexts, to discuss cloaking. Note, however, that for an analytic solution to exist, the exponents for the transverse and radial components must be the same.

We note in addition that Lucas et al. [55] have suggested radially anisotropic graphitic shells in the context of studies of fullerenes. We also note that we ourselves have also earlier treated particular examples of such systems. The optics of polymer dispersed liquid crystal films (PDLC), tunable composites consisting of liquid crystalline droplets in an isotropic matrix, have been discussed by some of the present authors in previous work [56,57]. Shelestiuk et al. [19] have derived an effective medium theory for the dielectric properties of a colloid consisting of ferroelectric particles suspended in a nematic liquid crystal, with the aim of deriving important liquid crystal properties. In work more directly related to the core-shell models discussed in this paper, Kiselev et al. [58] have developed a $T$-matrix theory of scattering by radial and uniform anisotropic scatterers in an isotropic polymer matrix, although this paper stops short of discussing properties of media consisting of such particles.

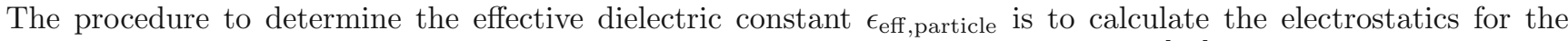
system, by solving $\nabla \cdot \boldsymbol{D}=0 ; \nabla \times \boldsymbol{E}=0$, subject to a given far field of magnitude $E_{0}[52]$. Outside the particle, the electrostatic potential takes the same form as it would, were the particle uniform inside [59]. The quantity $\epsilon_{\text {eff,particle }}$ can then be determined by noting the analogy with the formula for uniform spherical particles of radius $a$ and dielectric constant $\epsilon$. In this case, the external electrostatic potential $\Phi(r, \theta)$ is given by

$$
\Phi(r, \theta)=\left(\frac{a^{3}(\epsilon-1)}{\epsilon+2} r^{-2}-r\right) E_{0} \cos \theta .
$$

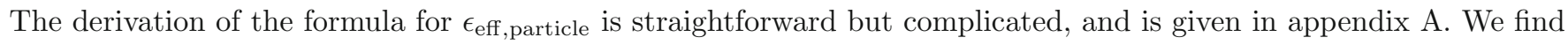

$$
\epsilon_{\text {eff }, \text { particle }}=\epsilon_{\|, 0} \frac{t_{2}\left[t_{1} \frac{\epsilon_{\|, 0}}{\epsilon_{c}}\left(\frac{b}{a}\right)^{m}-1\right]\left(\frac{b}{a}\right)^{\xi}-t_{1}\left[t_{2} \frac{\epsilon_{\|, 0}}{\epsilon_{c}}\left(\frac{b}{a}\right)^{m}-1\right]}{\left[t_{1} \frac{\epsilon_{\|, 0}}{\epsilon_{c}}\left(\frac{b}{a}\right)^{m}-1\right]\left(\frac{b}{a}\right)^{\xi}-\left[t_{2} \frac{\epsilon_{\|, 0}}{\epsilon_{c}}\left(\frac{b}{a}\right)^{m}-1\right]},
$$

where $t_{1}, t_{2}$ and $\xi$ are algebraic functions of $m$ and $\epsilon_{\perp, 0} / \epsilon_{\|, 0}$ defined in appendix A.

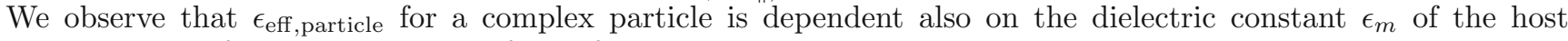
medium, whereas of course in the case of a uniform particle the particle dielectric constant is given. In the case $m=0$, eq. (4) reduces to the result given by Kettunen et al. [59]. We remark that a simple Born approximation calculation of the scattering amplitude of the core-shell nanoparticle would simply average the dielectric constant inside the whole complex nanoparticle. In fig. 4 we show some comparisons of the effective shell-core dielectric constant with that calculated using naive averaging over shell volume. The two calculations differ dramatically, and show convincingly the importance of calculating the internal fields in a sufficiently sophisticated manner.

In the context of determining the effective dielectric constant of a system in which such particles are embedded in a host, this is a necessary first step to average over the local structure. In refs. [59,60] it has been labelled "internal 


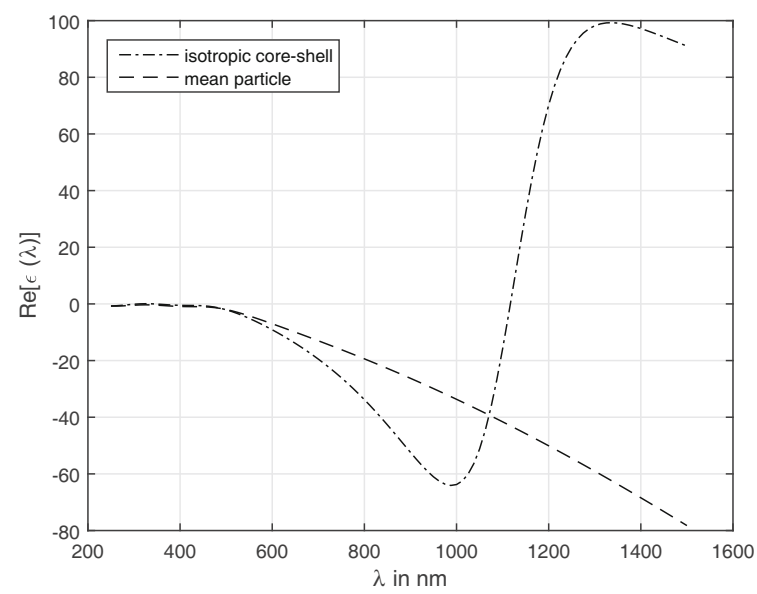

(a)

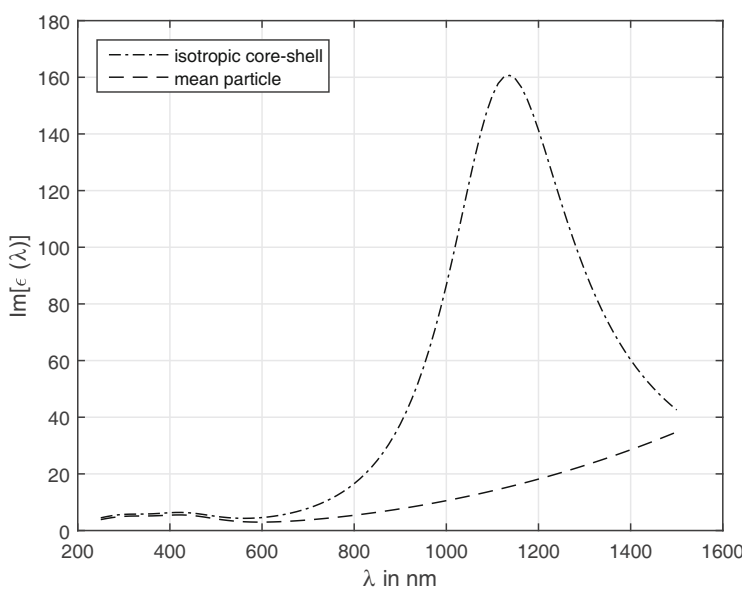

(b)

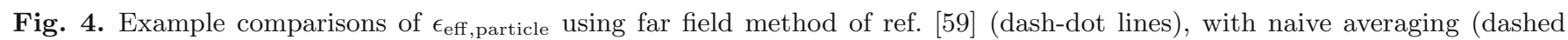
lines): (a) $\operatorname{Re}(\epsilon)$; (b) $\operatorname{Im}(\epsilon)$. Naive averaging fails completely to pick up the plasmonic resonance.

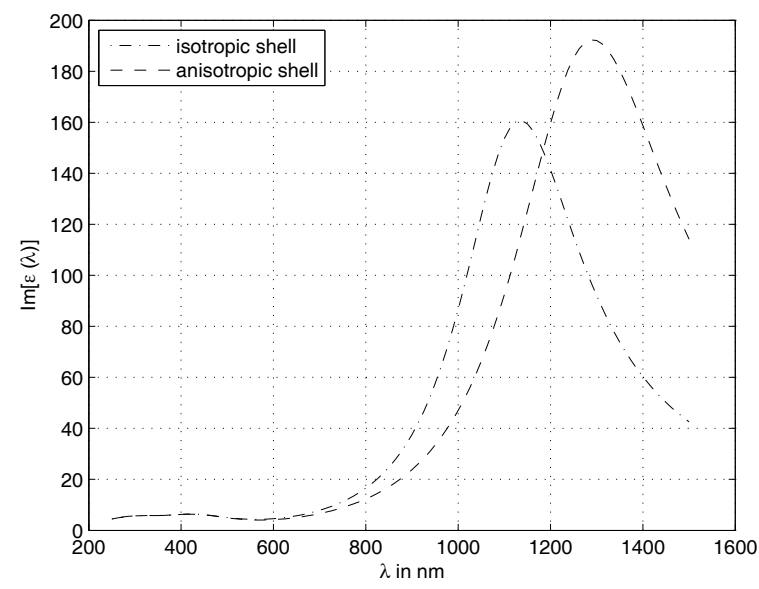

(a)

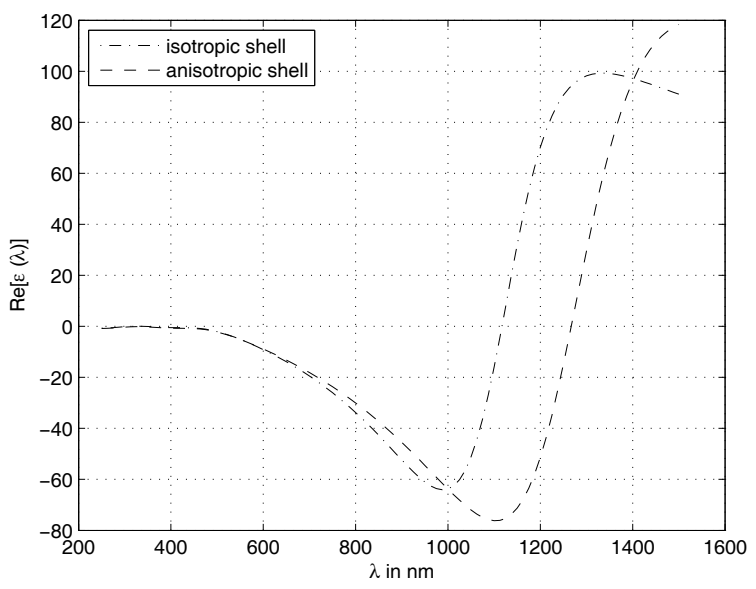

(b)

Fig. 5. Comparison of effective dielectric functions of particles as a function of wavelength, with isotropic (dot-dash lines) and uniform but radially anisotropic (dash lines) shells. Imaginary (a), and real (b), parts of the effective dielectric function $\epsilon(\lambda)$ of total core-shell particle. Core $\mathrm{Au}$, radius $3 \mathrm{~nm}$, with wavelength-dependent properties from fig. 1 . Core volume fraction: 0.85 (shell radius $\sim 3.167 \mathrm{~nm}$ ). Plausible example values of shell dielectric taken in such a way that the trace of the permittivity in both cases is the same: shell $\epsilon=3$, anisotropic shell $\epsilon_{r}=4, \epsilon_{t}=2.5$.

homogenization". An analogous procedure has also been applied to infinitely long cylinders, in which the axis of the cylinder is perpendicular to the incident light. In this case, the effective dielectric constant is now given by

$$
\epsilon_{\text {eff }, \text { particle }}=\epsilon_{\|, 0} \frac{s_{2}\left[s_{1} \frac{\epsilon_{\|, 0}}{\epsilon_{c}}\left(\frac{b}{a}\right)^{m}-1\right]\left(\frac{b}{a}\right)^{\psi}-s_{1}\left[s_{2} \frac{\epsilon_{\|, 0}}{\epsilon_{c}}\left(\frac{b}{a}\right)^{m}-1\right]}{\left[s_{1} \frac{\epsilon_{\|, 0}}{\epsilon_{c}}\left(\frac{b}{a}\right)^{m}-1\right]\left(\frac{b}{a}\right)^{\psi}-\left[s_{2} \frac{\epsilon_{\|, 0}}{\epsilon_{c}}\left(\frac{b}{a}\right)^{m}-1\right]},
$$

where $s_{1}, s_{2}$ and $\psi$ are algebraic functions of $m$, and $\epsilon_{\perp, 0} / \epsilon_{\|, 0}$ defined in appendix B.

We now present some numerical examples which illustrate the principles we have discussed above. In fig. 5 we examine wavelength dependence of the real and imaginary parts of internally homogenized dielectric functions of a particle with an $\mathrm{Au}$ shell surrounded by a homogeneous dielectric shell, where apart from Au plasmon effects, the optics only assumes a quasi-static limit. This figure compares the effects of an isotropic shell with that of a significantly anisotropic shell (but with the anisotropy in the radial direction, as discussed above) with the same mean permittivity. In this case, the effect of radially anisotropic shell is to shift features of the dielectric spectrum to higher wavelengths, while exaggerating extrema. An interesting question concerns which component of the permittivity is the most important, and whether a comparison which conserved transverse (or longitudinal) permittivity components, for example, would be more physically revealing. We postpone this question to further work. 


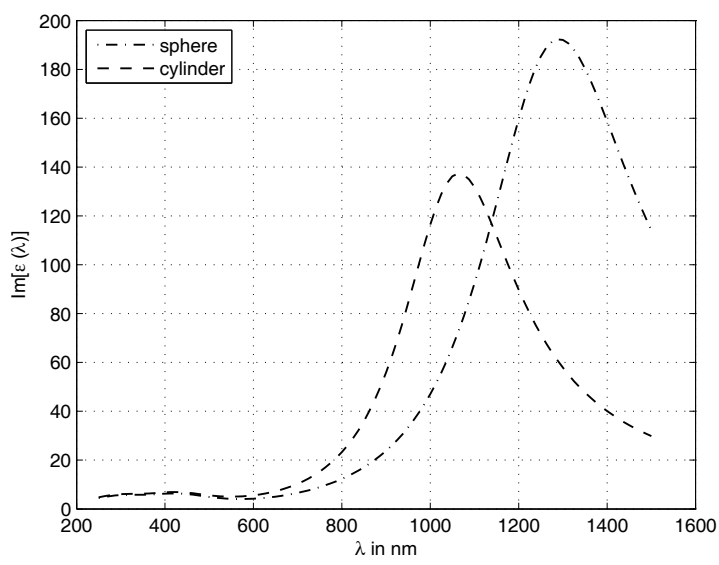

(a)

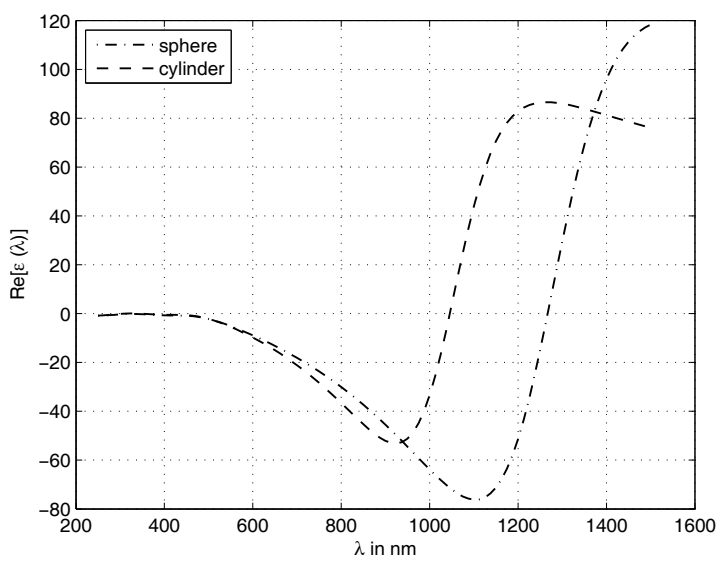

(b)

Fig. 6. Comparison of effective dielectric functions of spherical and cylindrical particles, with anisotropic shells. In each case, core radius $3 \mathrm{~nm}$, filling factor 0.85 . isotropic (dot-dash lines) and radially anisotropic (dash lines) shells. Imaginary (a) and real parts (b) of the effective dielectric function $\epsilon(\lambda)$ of total core-shell particle. Core Au with wavelength-dependent properties from fig. 1. Anisotropic shell $\epsilon_{r}=4, \epsilon_{t}=2.5$.

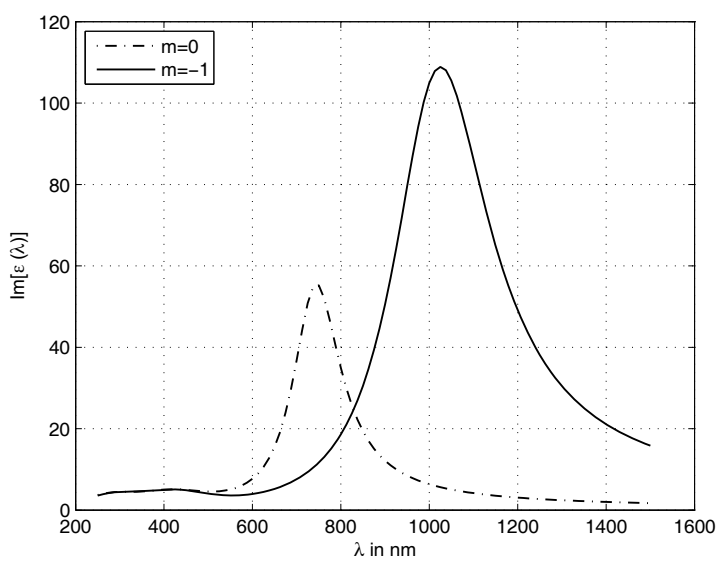

(a)

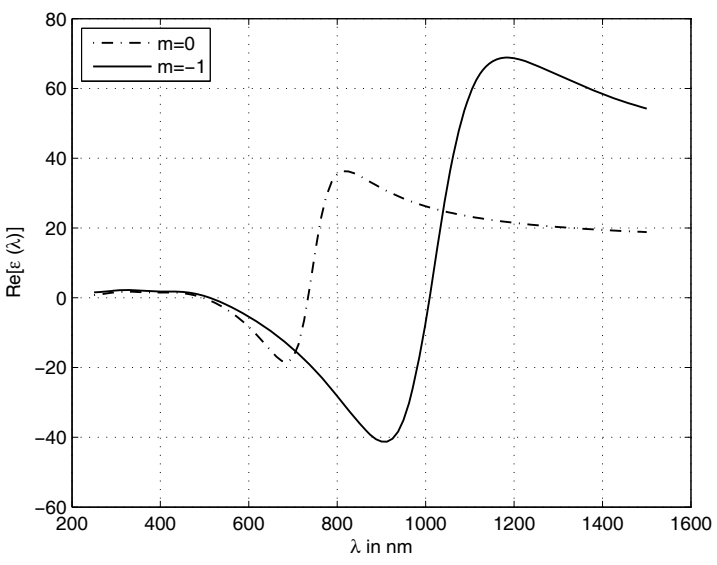

(b)

Fig. 7. Comparison of homogeneous (dot-dash line) and inhomogeneous (full line) shells. Both shells with radial anisotropy. Core properties as in from fig. 1. Core radius $3 \mathrm{~nm}$, filling factor 0.5. $m=0$ (homogeneous shell) $\epsilon_{r}=4, \epsilon_{t}=2.5$. Mean dielectric properties in inhomogeneous $(m=-1)$ and homogeneous $(m=0)$ shells identical.

We then pose the question whether the dielectric response of a cylinder or a sphere is larger. These results are plotted in fig. 6 . Here we retain a radially anisotropic shell in both cases. Our treatment of the cyclinder electrostatics supposes that the electric field polarization is perpendicular to the cylinder axis. This can correspond either to light incident along the cylinder axis, or alternatively to polarized light incident at some other angle to the axis. In order to provide a sensible comparison, we do not keep the internal and external radii the same, but rather suppose that the cores possess the same radii, and filling fractions. Thus the proportion of the complex particle consisting of Au core is the same in the two cases. Again we have plotted wavelength dependence of the real and imaginary parts of the dielectric function. We observe that changing the shape of a complex particle with otherwise identical quantitative properties can noticeably change its optical properties. We speculate that a sphere and a cylinder are two limiting cases of extreme shapes, and that other regular shapes might have intermediate properties.

In fig. 7 we compare the properties of particles with homogeneous and inhomogeneous anisotropic shells. The two systems are chosen to possess the same average dielectric behaviour, so that naive averaging would imply identical mean dielectric properties. But in fact, although qualitatively similar, the plots exhibit significant quantitative differences, both in terms of the position and the magnitude of resonances. The inhomogeneous shell has a significantly sharper and more exaggerated wavelength dependence for both real and imaginary parts of the internally homogenized permittivity, and also the resonance is shifted by a factor of approximately 1.3 . 


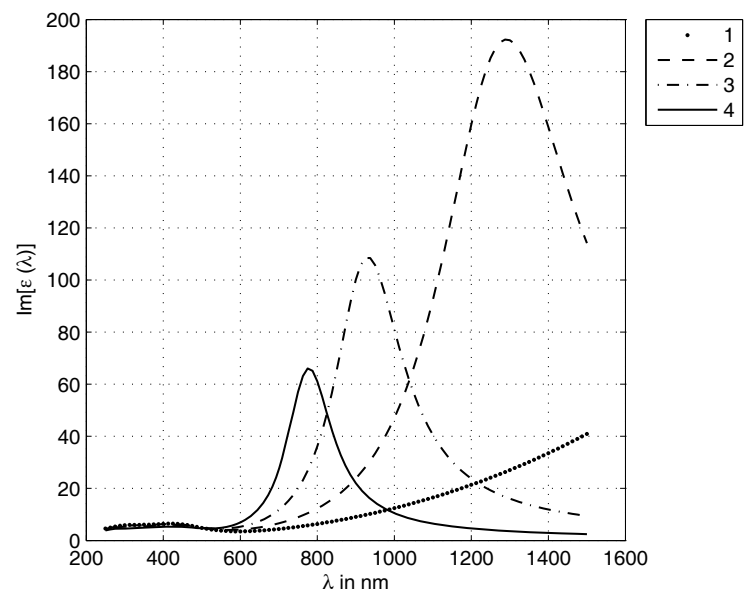

(a)

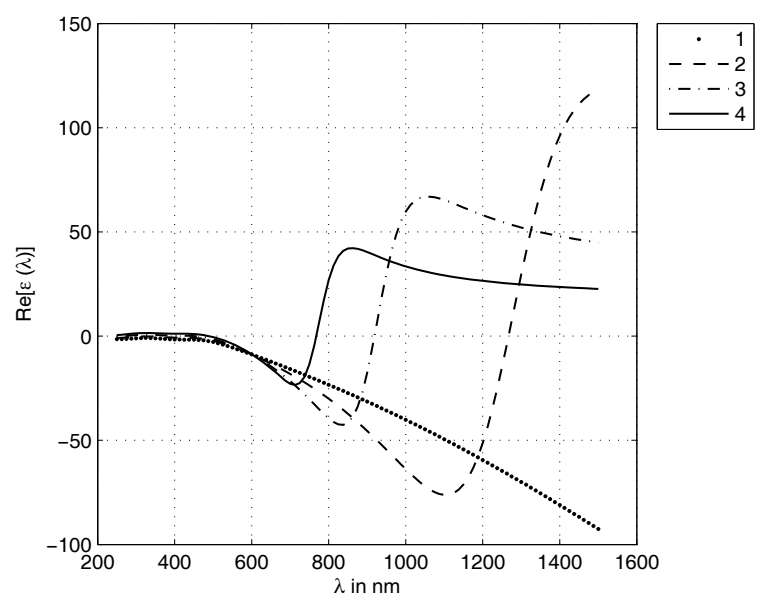

(b)

Fig. 8. Effect of filling factor $f$ on internally homogenized dielectric function; (a) imaginary part, (b) real part. In all cases, spherical Au core radius $3 \mathrm{~nm} . m=0$ (homogeneous shell), and shell radially anisotropic: $\epsilon_{r}=4, \epsilon_{t}=2.5$. Dotted line $(1) f=1$ (no shell); dashed line (2) $f=0.85$; dash-dot line $f=0.7$; full line (4) $f=0.55$.

In fig. 8, we show the effect of increasing the shell radius (or equivalently of decreasing the filling factor $f$ ) on the internally homogenized dielectric function, starting with no shell at all, and finishing with a shell radius a factor of 1.22 larger than that of the core. We observe that the absorption resonance associated with the peak in the imaginary part of the internally homogenized dielectric function occurs at increasingly reduced wavelengths (or increased frequencies) as the shell thickness is increased, and that even relatively small changes in the shell thickness change the absorption spectrum significantly. The absorption peak corresponds to a relatively sharp increase in the real part of the permittivity.

\section{Ensembles of particles}

In general, to discuss the average properties of composites in which uniform spherical particles are embedded in an anisotropic host, it is necessary to use an anisotropic effective medium theory. For a Maxwell-Garnett-type [19, 20] theory, Sihvola and coworkers [20,21] have derived the following formulas for the effective dielectric tensor of a system of spherical inclusions of volume fraction $\eta$ with dielectric constant $\epsilon_{i}$ embedded in an anisotropic dielectric with principal components $\epsilon_{\|}=c \epsilon_{\perp}$ :

$$
\begin{gathered}
\epsilon_{\mathrm{eff}, \|}=\epsilon_{\|}+\eta \frac{\epsilon_{\|}\left(\epsilon_{i}-\epsilon_{\|}\right)}{\epsilon_{\|}+(1-f) L_{\|}\left(\epsilon_{i}-\epsilon_{\|}\right)}, \\
\epsilon_{\mathrm{eff}, \perp}=\epsilon_{\perp}+\eta \frac{\epsilon_{\perp}\left(\epsilon_{i}-\epsilon_{\perp}\right)}{\epsilon_{\perp}+(1-f) L_{\perp}\left(\epsilon_{i}-\epsilon_{\perp}\right)} .
\end{gathered}
$$

The quantities $L_{\|}, L_{\perp}$ are depolarization factors for spheroids:

$$
\begin{aligned}
L_{\|} & =\frac{c}{c-1}\left(1-\frac{\tan ^{-1} \sqrt{c-1}}{\sqrt{c-1}}\right), \\
L_{\perp} & =\frac{1}{2(c-1)}\left(1-c \frac{\tan ^{-1} \sqrt{c-1}}{\sqrt{c-1}}\right),
\end{aligned}
$$

with $L_{\|}+2 L_{\perp}=1$. The formulas suppose that the host is uniaxially anisotropic, and inclusion is spherical. In principle it is possible to treat the general case, but the formulae become more complicated, and less analytically tractable.

To apply this theory to the case of core-shell spherical inclusions, we shall now use the internally homogenized dielectric parameters of the core-shell nanoparticles. The value of $\epsilon_{\text {eff particle }}$ chosen is determined supposing the particle to be embedded in an isotropic medium with an appropriate mean dielectric constant $\epsilon_{m}=\frac{\epsilon_{\|}+2 \epsilon_{\perp}}{3}$. In the optical regime, in which the anisotropy is weak $\left(\frac{\epsilon_{\|}-\epsilon_{\perp}}{\epsilon_{m}} \sim 0.1\right)$, we expect this approximation, while uncontrolled, to capture the basic physics. In appendix $\mathrm{C}$, we address the reliability of this approximation by using commercial software to solve Maxwell's equations in the region of the core-shell particle exactly. We find that the approximation is indeed good. 


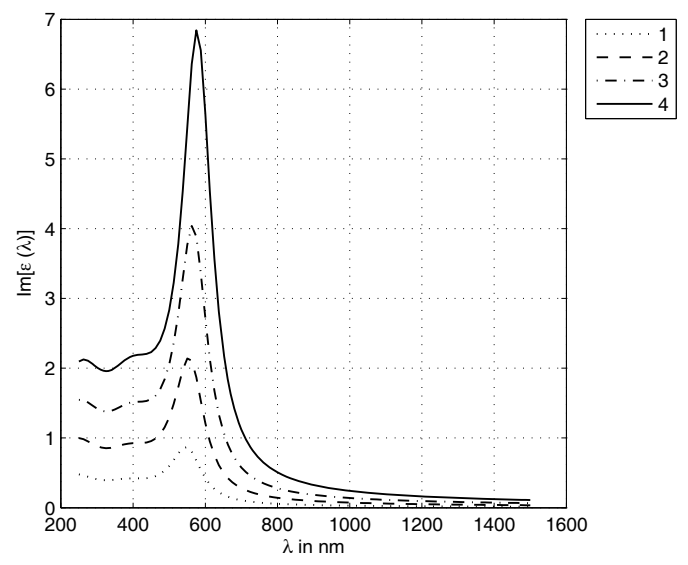

(a)

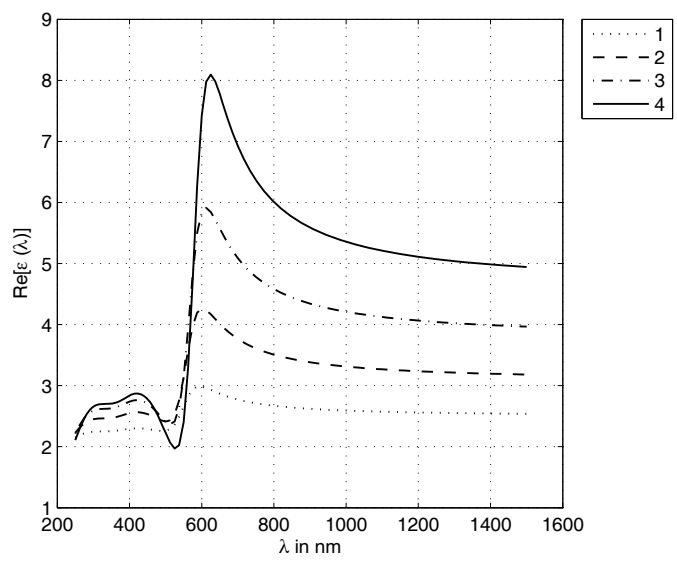

(b)

Fig. 9. Spherical anisotropic core-shell particles in isotropic host medium. Wavelength dependence of effective dielectric parameters of colloidal suspensions consisting of spherical anisotropic core-shell particle in isotropic host. As in previous figures, Au core: $R_{\text {core }}=3 \mathrm{~nm}$; dielectric shell $\epsilon_{r \text { shell }}=4 ; \epsilon_{t \text { shell }}=2.5 ; f=0.7$, with host dielectric $\epsilon_{\text {host }}=2$. Curves $1,2,3,4$ correspond respectively to volume fractions $\eta=0.1,0.2,0.3,0.4$.

A similar philosophy can be applied to discuss systems in which the spheres are replaced by cylinders, although the problem is significantly more complicated mathematically. The orientation of the cylinders is now relevant, because the optical axes of the host and of the cylinder do not necessarily coincide. Fortunately there are some easily accessible limits, namely when the cylinders are aligned: a) along the bulk easy axis; b) perpendicular to it; and c) when they are randomly aligned in an isotropic host.

In cases a) and b), we may use formulas (29) in ref. [19] (See appendix for a discussion of depolarization effects associated with anisotropic inclusions in anisotropic materials.). In case c), we can employ formulas introduced by $\mathrm{Yu}$ and Gao [61], who have discussed a composite with embedded randomly oriented cylinders. Sihvola [21] gives the following formulas for cylinders aligned along the $O Z$-axis, with isotropic dielectric properties $\epsilon_{i}$ in the $x y$-plane; the anisotropic principal host has dielectric axes in the $x, y$ directions $\epsilon_{x} \neq \epsilon_{y}$. Then the effective medium dielectric properties are given by

$$
\epsilon_{\mathrm{eff}, K}=\epsilon_{K}+\eta \frac{\epsilon_{K}\left(\epsilon_{i}-\epsilon_{K}\right)}{\epsilon_{K}+(1-f) L_{K}\left(\epsilon_{i}-\epsilon_{K}\right)}
$$

with $K=x, y$, and the depolarization factors given by

$$
L_{y}=\frac{\sqrt{c}}{1+\sqrt{c}} ; \quad L_{x}=\frac{1}{1+\sqrt{c}} ; \quad c=\frac{\epsilon_{x}}{\epsilon_{y}} .
$$

\section{Numerical examples}

We now present some specific numerical examples to show how these effects translate into the effective medium properties of colloids with core-shell particle inclusions. The first example, shown in fig. 9, concerns a set of colloids in which spherical anisotropic core-shell particles are suspended in an isotropic host. As expected the imaginary part of the permittivity increases monotonically with particle volume fraction. For the real part, however, the most noticeable feature, apart from the increase in value, is that the absorption edge associated with the plasmonic peak is red-shifted by some $5 \%$ in wavelength at the highest volume fractions.

We now turn to radially anisotropic core-shell systems embedded in an anisotropic host medium. In this case calculation of both the parallel and tranverse components of the effective medium is required, using eqs. (6). In the anisotropic host medium, features similar to those in the isotropic host medium are seen, viz. both the parallel and perpendicular components of the permittivity increase in proportion to the colloidal concentration, together with small shifts in the plasmonic absorption edge, as shown in fig. 10, in which results are shown for different volume fractions at constant filling factor.

In fig. 11, rather than vary the colloidal volume fraction $\eta$, we fix the volume fraction, but vary the size of the core, by varying the filling factor $f$. The broadest absorption peak is seen to occur when there is no shell. This might be expected, in that the absorption is due to the metallic core. Reducing $f$ involves progressively replacing metallic core by dielectric shell, which we expect to reduce both the magnitude and the width of the absorption peak, fig. 12 . 


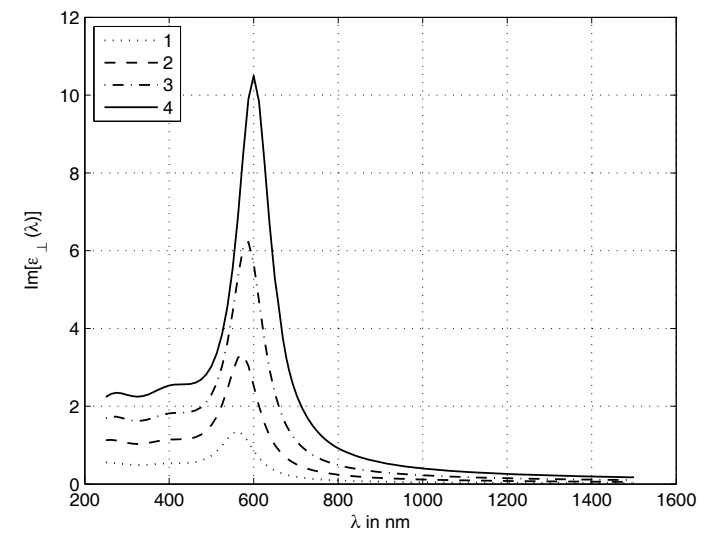

(a)

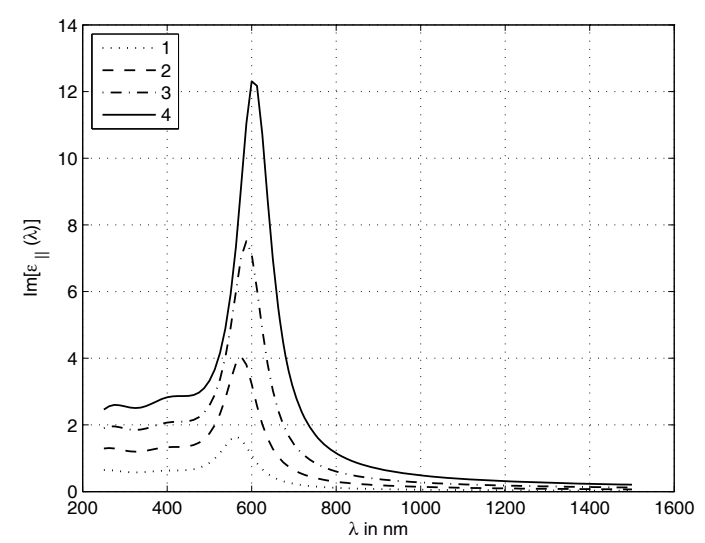

(c)

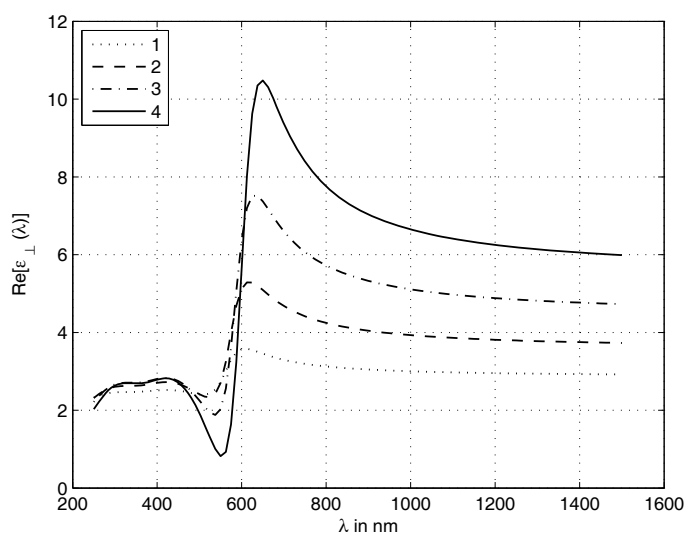

(b)

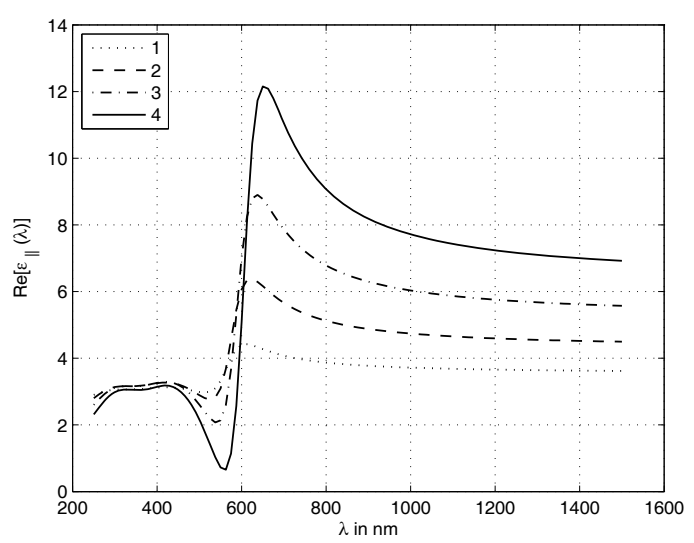

(d)

Fig. 10. Spherical anisotropic core-shell particles in an anisotropic host medium. Wavelength dependence of effective dielectric parameters of colloidal suspensions consisting of spherical anisotropic core-shell particles in an anisotropic host, for different values of volume fraction $\eta$ at constant particle shell filling fraction $f=0.7$. Plots $(\mathrm{a}, \mathrm{b})$ : real and imaginary parts of permittivity of effective $\epsilon_{\perp \text { medium }}$; curves (c, d): real and imaginary parts of effective $\epsilon_{\| \text {medium. }}$. Curves 1 (dots), 2 (dash), 3 (dot-dash) and 4 (full line) correspond respectively to volume fractions $\eta=0.1,0.2,0.3,0.4$. Au core: $R_{\text {core }}=3 \mathrm{~nm}$; dielectric shell $\epsilon_{r \text { shell }}=4 ; \epsilon_{t}$ shell $=$ 2.5, while the host properties are $\epsilon_{\|}=1.7^{2}, \epsilon_{\perp}=1.5^{2}$, corresponding to the optical regime of a typical nematic liquid crystal.

A more detailed understanding of the difference between the two cases requires further analysis, but the sets of curves are sufficiently different to assert conclusively that shape does matter.

Our final example concerns hyperbolic media, which have attracted considerable recent interest [16]. We observe that to construct a hyperbolic medium, it is only sufficient to include suitable inclusions in an anisotropic host at a suitable volume fraction [62]. In ref. [62], however, the structure and metamaterial properties are usually fixed, but if the inclusion properties are tunable, so will the effective properties of the metamaterial [63]. In figs. 13 we demonstrate how transforming the shell of an inclusion from an anisotropic to an equivalent isotropic medium can switch the medium from hyperbolic to normal behavior. In the case studied in figs. 13, this occurs only in a very narrow frequency range. But we note that the parameters have not been optimised, and seek here only to point out the existence of this switching phenomenon.

\section{Inverted systems}

A number of authors [64-66] have discussed what might be regarded as inverted core-shell particles, in which by contrast with the materials discussed so far in this article, the dielectric material is in the core, but the shell is metallic. The methods we have developed in this paper can also be applied to materials including such nanoparticles. 


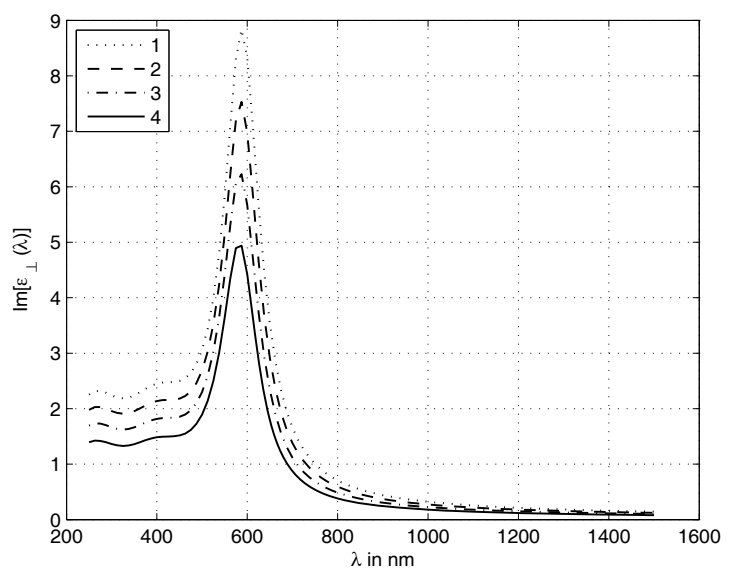

(a)

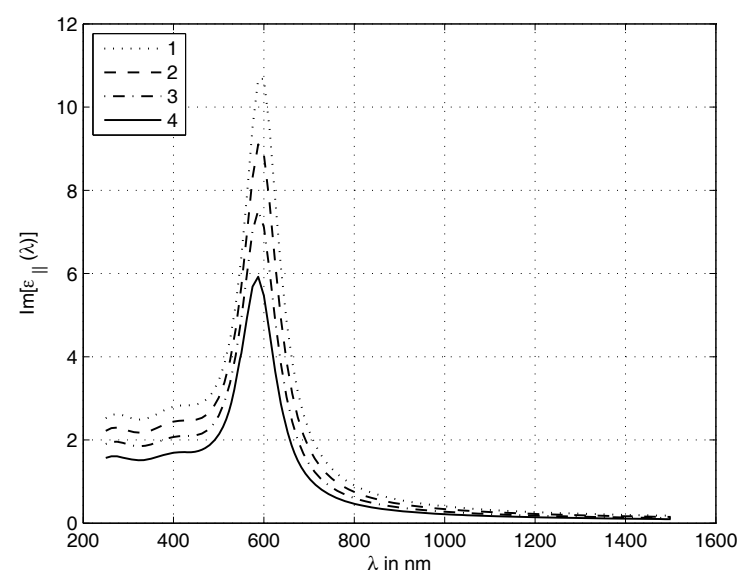

(c)

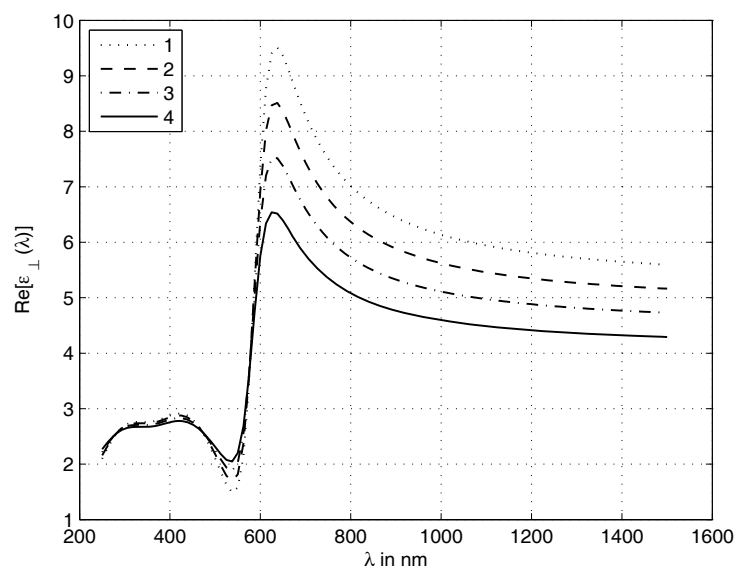

(b)

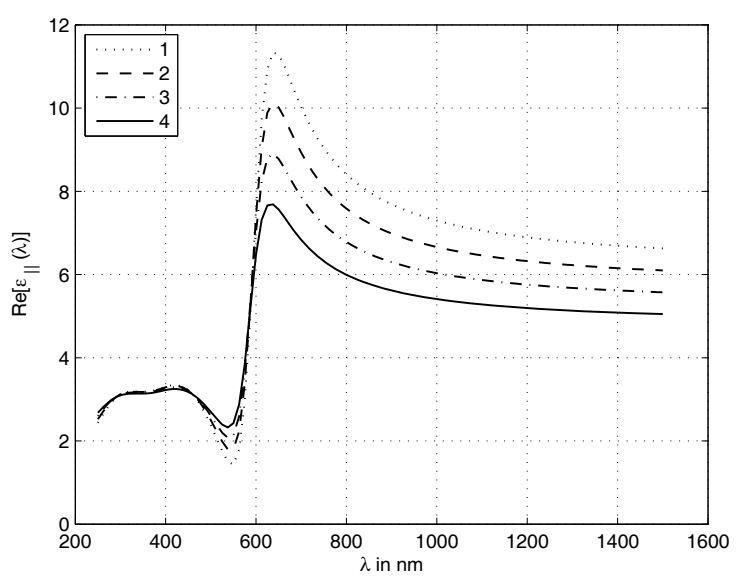

(d)

Fig. 11. Spherical anisotropic core-shell particles in an anisotropic host medium. Wavelength dependence of effective dielectric parameters of colloidal suspensions consisting of spherical anisotropic core-shell particles in an anisotropic host, for different values of filling factor at constant particle volume fraction $\eta=0.3$. Plots $(\mathrm{a}, \mathrm{b})$ : real and imaginary parts of permittivity of

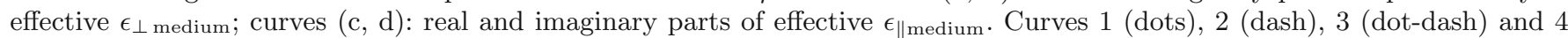
(full line) correspond respectively to filling fractions $f=1,0.85,0.7,0.55$. Au core: $R_{\text {core }}=3 \mathrm{~nm}$; dielectric shell $\epsilon_{r \text { shell }}=4$; $\epsilon_{t \text { shell }}=2.5$; while the host properties are $\epsilon_{\|}=1.7^{2}, \epsilon_{\perp}=1.5^{2}$, corresponding to the optical regime of a typical nematic liquid crystal.

In particular, the structure of the shell now becomes a critical determinant of the total effective medium permittivity. Depending on the details of the chemical process by which the particles, are synthesized, the metallic shell might consist either of solid continuous metal, or rather of a set of tiny metallic balls with the gaps between the balls filled with solvent [66]. The contrast between these two pictures is shown schematically in fig. 14 .

In the latter case, the electron mean free path would be limited by the size of the tiny balls, rather than, as might be expected, by the size of the shell. The consequence would be that the effective medium properties of the heterogeneous shell could differ greatly from those of a homogenous metallic shell. This would lead in turn to nanoparticle systems with unexpected total effective medium properties. We show the results of representative calculations, which show the key physical effect to which we wish to draw attention, in figs. 15 and 16.

In fig. 15 we compare results of a Mie scattering calculation of the wavelength dependence of the total cross-section of complex particles either with a dielectric core and respectively a solid Au shell, or a shell consisting of smaller $\mathrm{Au}$ particles. The cross-section involves both scattering and absorption. These calculations correspond to the physical pictures shown in fig. 14. The difference in input to the two calculations lies primarily in the imaginary part of the dielectric of the $\mathrm{Au}$, as discussed in eqs. (2), although the electron mean free path $L_{\text {eff }}$, taken to be the particle size, also affects the real part of the permittivity. 


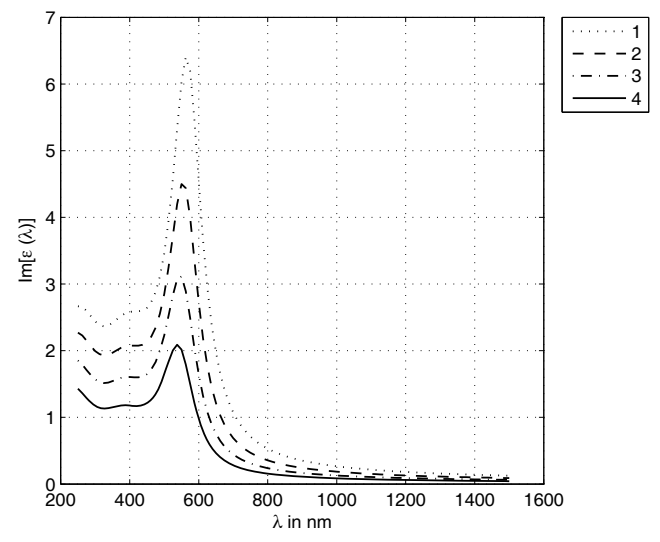

(a)

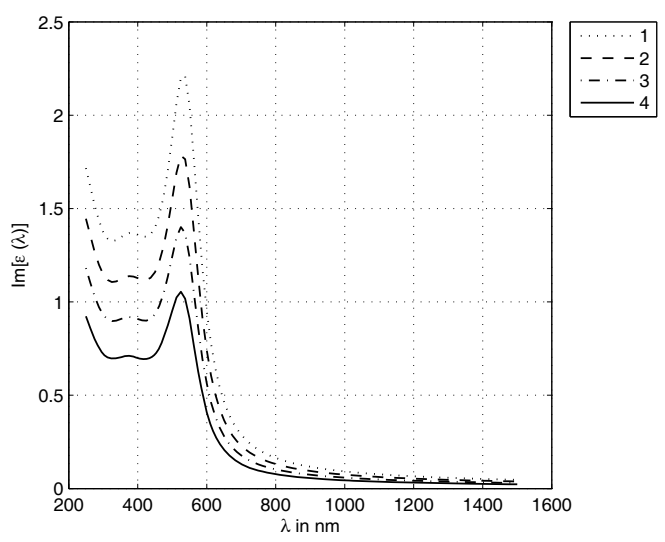

(c)

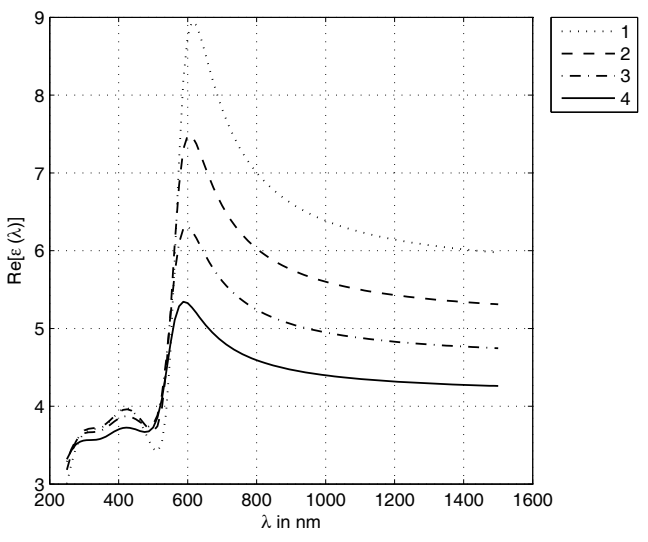

(b)

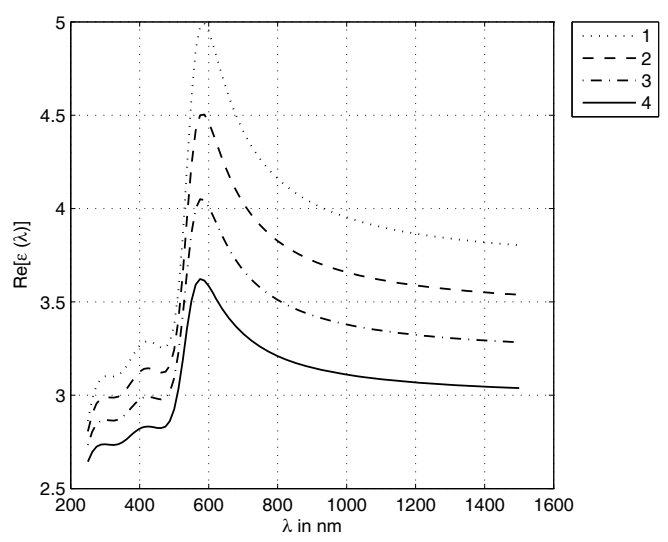

(d)

Fig. 12. Cylindrical anisotropic core-shell particles in an anisotropic host medium. Wavelength dependence of effective dielectric parameters of colloidal suspensions including cylindrical anisotropic core-shell particles in an anisotropic host. Au core: $R_{\text {core }}=$ $3 \mathrm{~nm}$; dielectric shell $\epsilon_{r \text { shell }}=1.7^{2} ; \epsilon_{t \text { shell }}=1.5^{2} ; \eta=0.7$. Host properties: $\epsilon_{\|}=1.7^{2}$, corresponding to the optical regime of a typical nematic liquid crystal. In each case, curves 1 (dots), 2 (dash), 3 (dot-dash) and 4 (full line) correspond respectively to filling fractions $f=1,0.85,0.7,0.55$. Curves $(\mathrm{a}, \mathrm{b})$ : imaginary and real parts of permittivity of effective $\epsilon_{\perp}$ medium, with host $\epsilon_{\perp}=2$. Curves (c, d): imaginary and real parts of effective $\epsilon_{\| \text {medium }}$ with host $\epsilon_{\perp}=3$.

The calculation builds on MATLAB software and algorithms developed by Mätzler [67], based on ref. [25], sect. 8.1. We suppose here that the total particle volume fraction is sufficiently small that there are no multiple scattering effects, so that a comparison with experiment can be effected simply by measuring the frequency dependence of light extinction (see, e.g., ref. [25], p. 69 and p. 287). On the other hand, the precise volume fraction of particles is always difficult to measure, and so absolute magnitudes would be difficult to compare experimentally. The really dramatic effects would rather be exhibited in the wavelength dependence of the extinction, and it is this quantity which we have plotted in fig. 15, normalized with respect to its maximum value. The figure shows that whereas the solid shell shows a very narrow distinct maximum as a function of wavelength, the heterogeneous shell yields a flatter spectrum with a much broader maximum. In fig. 16 we show real and imaginary parts of effective dielectric parameters for inverted spherical core-shell particles in an anisotropic host. We remark that changing the packing fraction and size of the spheres in the shell constitutes another way of tuning the properties of nanoparticles and composite effective media.

\section{Conclusions}

We have outlined a procedure to calculate the effective permittivity of an anisotropic medium which contains complex spherically or cylindrically symmetric inclusions. The inclusions contain an isotropic core surrounded by a non-uniform shell. The key feature of the shells is that they are non-uniformly radially anisotropic, so that even though the inclusions are optically anisotropic locally, they are isotropic globally. The calculations are carried out in the quasi-static limit, or equivalently the wavelength is much longer than the characteristic size of particle inclusions. Because the permittivity takes an algebraic form in the shell, the electrostatics is formally soluble. When material in the core or in the shell is 


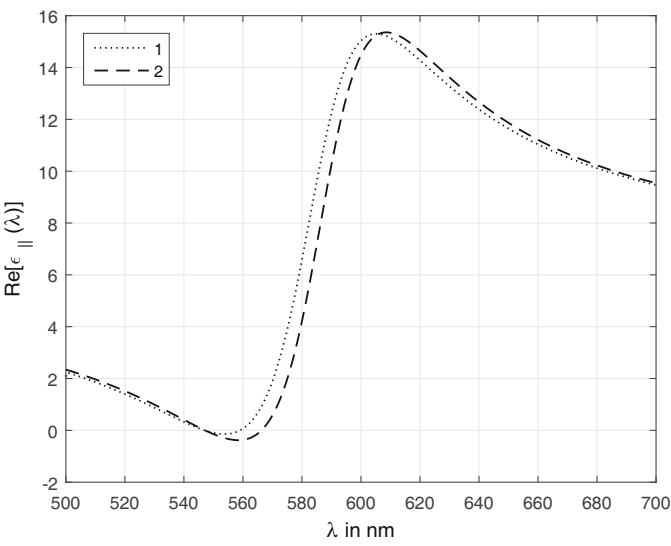

(a)

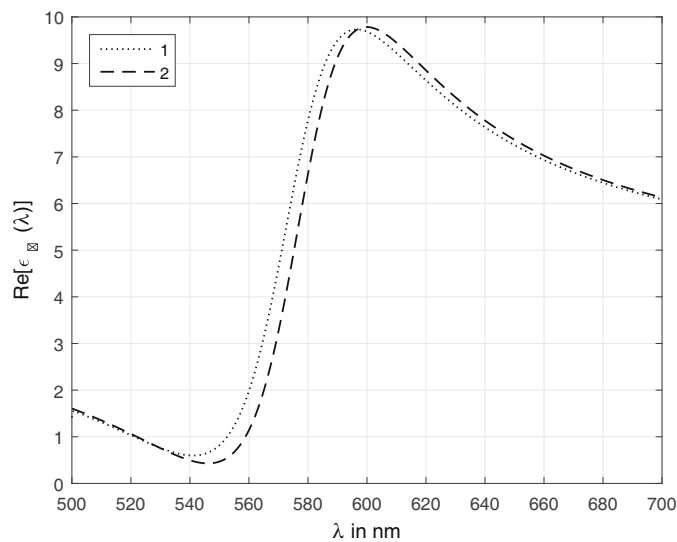

(c)

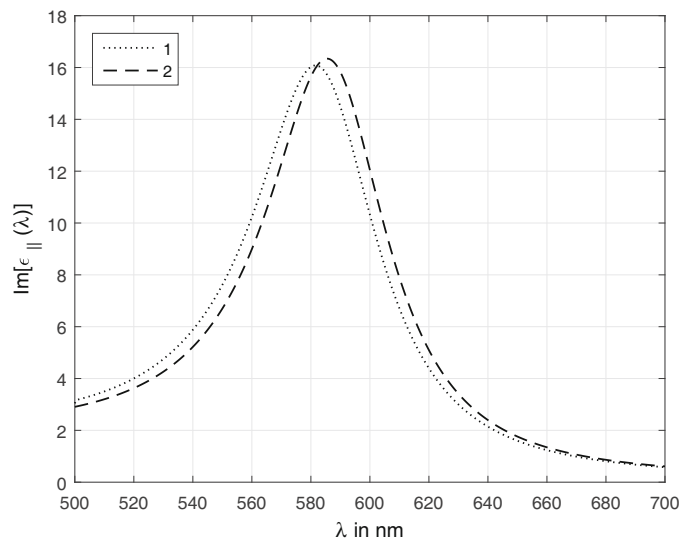

(b)

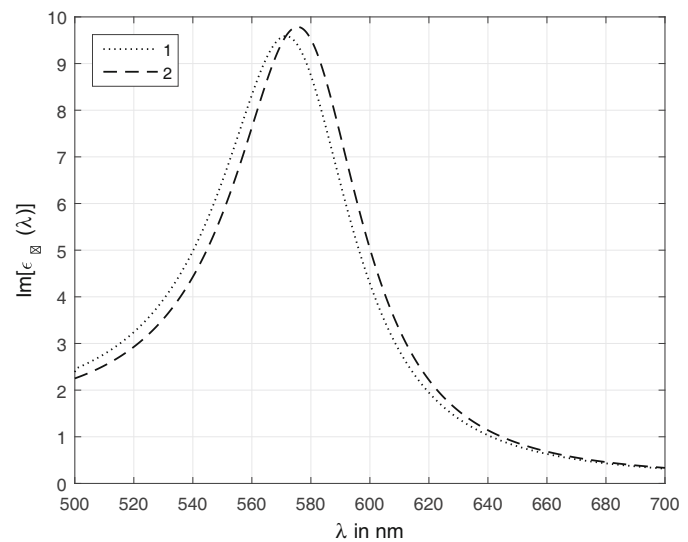

(d)

Fig. 13. Hyperbolic to anisotropic switching of spherical anisotropic core-shell particles in an anisotropic host medium. Wavelength dependence of effective dielectric parameters of colloidal suspensions consisting of spherical anisotropic core-shell particles. In all plots: Au core: $R_{\text {core }}=25 \mathrm{~nm}$. $f=0.75, \eta=0.3$ Host properties: $\epsilon_{\|}=3.61=1.9^{2}, \epsilon_{\perp}=1.96=1.4^{2}$ corresponding to the optical regime of a possible nematic liquid crystal. Dotted lines (curves 1) correspond to anisotropic dielectric shell: $\epsilon_{r \text { shell }}=5.0$ $\epsilon_{t \text { shell }}=2.0$. Dashed lines (curves 2 ) correspond to isotropic shell with $\epsilon=3.0$ (i.e. equivalent trace). Curves ( $\mathrm{a}$, b): Imaginary and real parts of effective $\epsilon_{\|}$. Curves (c, d): Imaginary and real parts of permittivity of effective $\epsilon_{\perp}$.
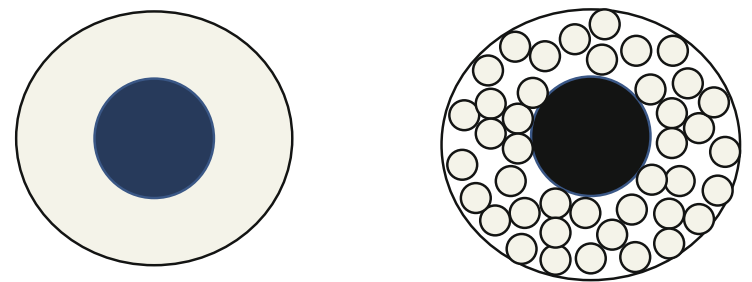

Fig. 14. Comparison of shells consisting of continuous metal (left) and metallic balls (right). Grey: metal. Black: dielectric core. White: solvent filler.

metallic, electron lifetimes in finite samples are reduced. The permittivity thus includes a size-dependent imaginary part, which must also be properly included in a full treatment. Although we include dielectric anisotropy, we neglect magnetic anisotropy because the particles are not magnetic. But if required in another case, the formalism allows extension to the case of diamagnetic anisotropy.

The electrostatics of the complex particle outside the inclusions is compared with that which follows from the electrostatics of a uniform particle. Using Clausius-Mossotti theory, the comparison yields explicit, if complicated, expressions for the effective dielectric properties of the complex particle. Finally, Maxwell-Garnett theory allows the medium and effective particle permittivities to be combined, to yield an effective permittivity for the whole system as a function of particle volume fraction. 


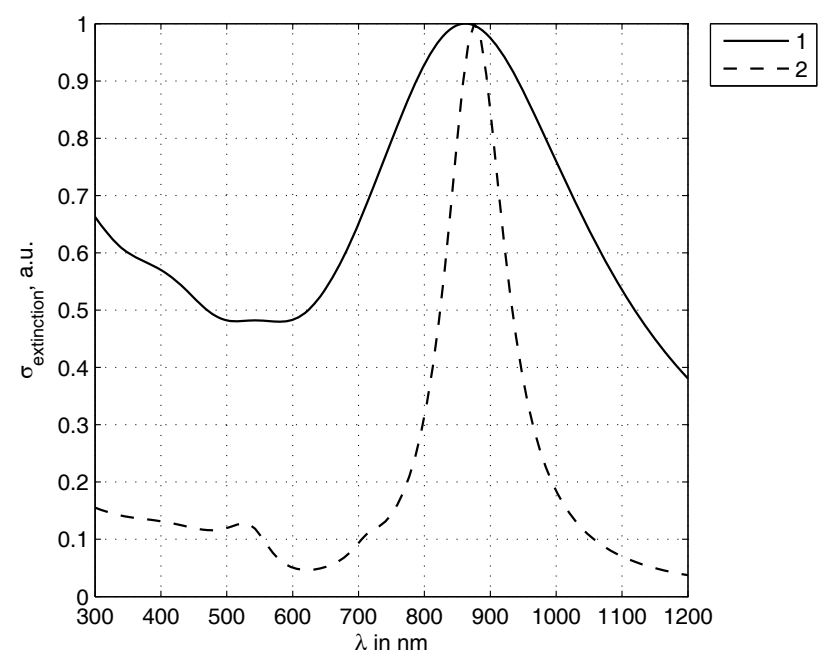

Fig. 15. Comparison of wavelength dependence of optical extinction properties for shells consisting of continuous metal (dashed line) and metallic balls (solid line), measured in arbitrary units. In each case, host solvent is water. Radius of silica core $20 \mathrm{~nm}$, refractive index $n=2.5$. Shell thickness: $10 \mathrm{~nm}$. In case (b) radius of small particles $=1 \mathrm{~nm}$; packing fraction (i.e. metallic particle volume fraction within the shell) $=0.74$ (i.e. close-packed spheres of equal size); solvent: water.

Core-shell particles are the focus of intense current interest because of their application to metamaterial systems. The anomalous electromagnetic properties of metamaterials give rise to numerous applications, and there is an enormous literature which it has been impossible to do justice here. From a physical point of view, our focus has been on the anisotropy of the host material, radial anisotropy of the shells, a more sophisticated treatment of the electronic properties inside the metallic core. As far as we are aware, this focus has so far been lacking in the literature.

Methodologically we have employed a Maxwell-Garnett type of method to calculate effective medium properties, using basic techniques which go back around a century. A particular difficulty has been that in order to make the calculation of the effective properties of the core-shell particle tractable, we have been forced to suppose that the host material is isotropic. In some sense the treatment is thus not entirely self-consistent. A deeper problem with the whole method is that it is heuristic. There is no small parameter allowing a limit in which we can say that the technique is exact. To make this method more sophisticated, two routes are possible.

One possible strategy involves refining the effective medium theory using spectral function techniques, following Bergman [2,31]. Lu et al. [68] have very recently used such a method for plasmonic nanocomposites with an isotropic shell and an isotropic host. It would be interesting to extend this method to the anisotropic case considered here, but the mathematics is not completely straightforward; we postpone this for further research.

The second strategy, involves exact solution of Maxwell's equations over a limited region. We have discussed possible difficulties of this approach in the Introduction. The usual procedure involves embedding a single-core-shell particle in a repeated box, although of course elaborations are possible. At least some workers in the field now regard this approach as having entirely replaced "old-fashioned" effective medium theories. Our view is that while "the singlecore-shell particle in a box" approach still contains approximations, in principle there is a computational route to macroscopic properties.

In sect. 4, we noted that the calculation of the effective properties of the core-shell particle supposing the host material to be isotropic is a weak link in our chain of reasoning. We compared our approximation to the single-coreshell particle approximation, solving Maxwell's equations exactly within the (repeating) box. A frequency-dependent comparison of the two sets of results showed them to be very similar. It is possible that in other physical contexts (e.g., if we understand it correctly, in [37]) this close matching will not hold. Further investigation would be fruitful as to the circumstances in which our effective medium approach is likely to be successful.

The methods we are proposing are computationally extremely cheap, easy to code, and in no way methodologically opaque. We particularly recommend them to experimentalists seeking insight into systems in which they may want to investigate a complicated parameter space. They can be regarded as complementary to computational methods which seek high accuracy and exact numbers, but involving greater computational difficulty.

Finally we repeat the most important findings of our study. The size of the metal core governs the electron mean free path, and hence affects the single-particle properties significantly (see fig. 1). Then, even though the inclusions are in some sense small, nevertheless the dielectric effects cannot be accounted for by naive averaging over the permittivity of the component parts, as one might expect if one were to apply the Born approximation (see fig. 4). Thus, even though the particles are small, their shape is important. A similar effect is seen when the particles have different shape but the same volume (see fig. 2). 


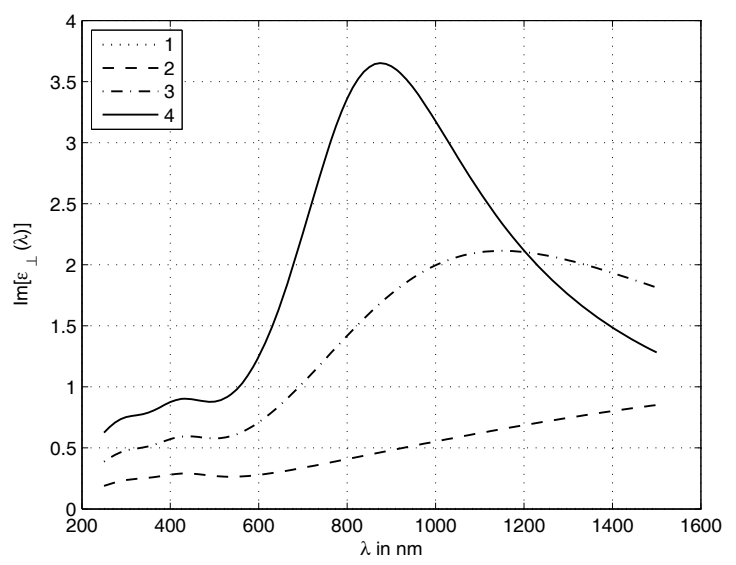

(a)

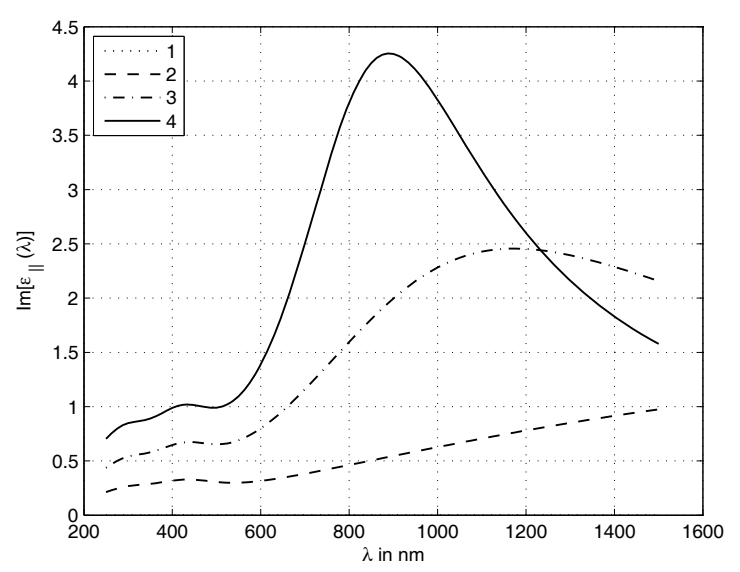

(c)

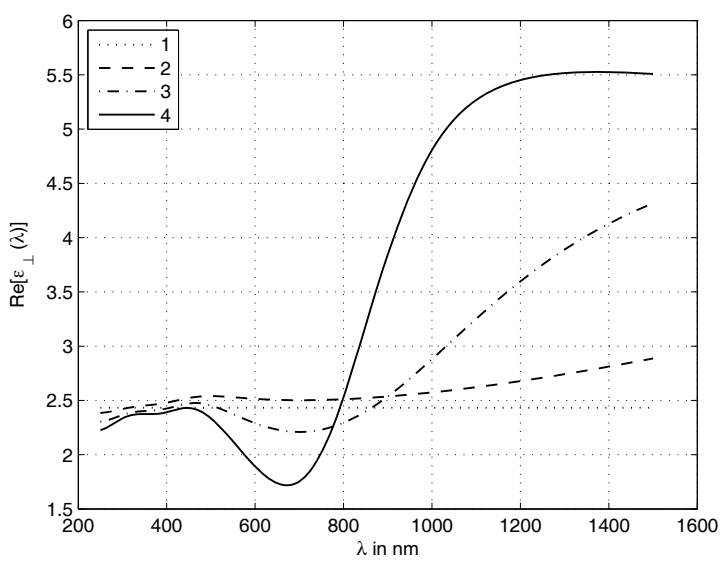

(b)

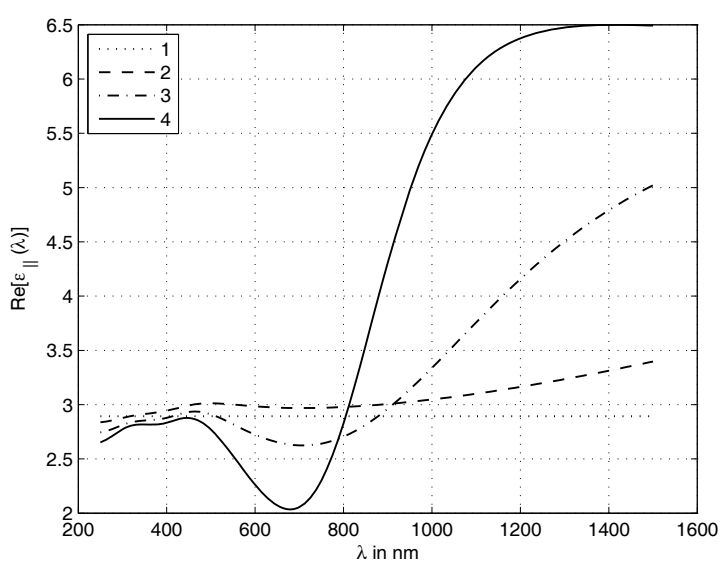

(d)

Fig. 16. Inverted spherical core-shell particles in an anisotropic host. Wavelength dependence of effective dielectric parameters of colloidal suspensions consisting of inverted spherical core-shell particles in an anisotropic host, as discussed in the text. In all cases, particle volume fraction $\eta=0.3$, Au shell: $R_{\text {core }}=3 \mathrm{~nm}$; anisotropic dielectric tensor of core $\epsilon_{r \text { core }}=4 ; \epsilon_{t}$ core $=2.5$; $f=0.7$, while the host properties are $\epsilon_{\|}=1.7^{2}, \epsilon_{\perp}=1.5^{2}$, corresponding to the optical regime of a typical nematic liquid crystal. Curves (a, b) (above): real and imaginary parts of permittivity of effective $\epsilon_{\perp}$ medium; curves (c, d) are real and imaginary

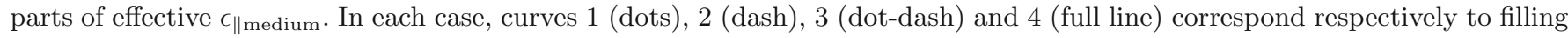
fractions $f=1,0.85,0.7,0.55$.

Likewise anisotropy is important; the prediction of the metamaterial effective coefficients are different depending on whether the particles contain a radially anisotropic shell or whether the shell is isotropic but with the same "average" permittivity (in the sense that in both cases the trace of the permitttivity tensor is the same, see fig. 6). We have shown an example in which, the isotropic inclusion allows, over a narrow range of wavelengths, an effective hyperbolic metamaterial. As no attempt was made to optimize parameters, it seems likely that with a suitably chosen parameter set, this will be quite a widespread phenomenon. We have also studied models for which the materials in the shell and the core are inverted, so that the shell consists of metal and the core is dielectric (see fig. 14). Now the mean free path in the metal again plays a significant role. In particular, if the shell consists of weakly coupled metallic balls, the mean free path is significantly reduced as compared to a continuous shell. In this case (see fig. 15), we predict that, as compared to the continuous shell, the absorption spectrum broadens significantly.

The key ideas of this work are as follows. First, inserting core-shell metal-dielectric nanoparticles into a host medium provides an opportunity to tune the effective dielectric function of the composite particle, and thus of the medium, by changing the properties of the core or the shell or both. Second, if the host is itself tunable, then this permits additional control of the effective dielectric function. In further work, we are extending this modelling strategy to include the possibility of a magneto-optic inhomogeneous core or shell. Finally we note that the optical and dielectric properties of the nanoparticle core or shell can also be tuned by pulse illumination. 
We acknowledge financial support from EOARD (grant 078001 to VYR and IPP), the hospitality of the University of Southampton (VYR), and useful discussions with Dr. Ron Ziolo (Centro de Investigación en Química Aplicada, Mexico), as well as with Dr. Sergey Basun (AFRL, USA). TJS acknowledges support from Azimuth Corporation, USA. VYR is particularly grateful to Drs. Silvia Irusta and Manuel Arruebo Gordo (Zaragoza, Spain) for useful conversations concerning the internal structure of the metallic shells of nanoparticles.

\section{Appendix A. Electrostatic formulae for spherical inclusions}

We suppose that an external field $E_{0}$ is applied in the $z$-direction. We consider a sphere of radius $b$, placed inside an annulus of radius $a$ which surrounds it. The geometry was given in fig. 3 above. The field inside the inclusion is given by $\mathbf{E}=-\nabla \boldsymbol{\Phi}$. For a radially symmetric dielectric we have radial and transverse principal components $\epsilon_{r}(r)=\epsilon_{\|}(r)$, and $\epsilon_{t}(r)=\epsilon_{\perp}(r)$. We use polar coordinates $(r, \theta, \phi)$, but the cylindrical symmetry imposed by the external field in the $z$-direction implies that all derivatives in the $\phi$ direction are zero and we ignore this coordinate

The electric displacement $\mathbf{D}$ is given by

$$
\mathbf{D}=\epsilon_{\|} \frac{\partial \Phi}{\partial r} \mathbf{e}_{r}+\epsilon_{\perp} \frac{1}{r} \frac{\partial \Phi}{\partial \theta} \mathbf{e}_{\theta}
$$

In the absence of charges, the governing equation is $\nabla \cdot \mathbf{D}=0$, which in polar coordinates reads:

$$
\nabla \cdot \mathbf{D}=\frac{1}{r^{2}} \frac{\partial}{\partial r}\left(r^{2} \epsilon_{\|} \frac{\partial \Phi}{\partial r}\right)+\frac{1}{r \sin \theta} \frac{\partial}{\partial \theta}\left(\sin \theta \epsilon_{\|} \frac{1}{r} \frac{\partial \Phi}{\partial r}\right)=0 .
$$

The radial symmetry of the inclusions imposes that $\epsilon_{i}(\mathbf{r})=\epsilon_{i}(r)$, where we assume subscripts $i=\|, \perp$. Separation of variables implies a solution of the form

$$
\Phi(r, \theta)=f(r) \cos \theta .
$$

Applying this formula to eq. (A.2) yields

$$
\nabla \cdot \mathbf{D}=\frac{\epsilon_{\|}}{r^{2}} \cos \theta\left[r^{2} \frac{\partial^{2} f}{\partial r^{2}}+\frac{1}{\epsilon_{\|}} \frac{\partial \epsilon_{\|}}{\partial r} \frac{\partial f}{\partial r}+2 r \frac{\partial f}{\partial r}-\frac{2 \epsilon_{\perp}}{\epsilon_{\|}} f\right]=0,
$$

which implies that the quantity in the square bracket is also zero:

$$
r^{2} \frac{\partial^{2} f}{\partial r^{2}}+\frac{1}{\epsilon_{\|}} \frac{\partial \epsilon_{\|}}{\partial r} \frac{\partial f}{\partial r}+2 r \frac{\partial f}{\partial r}-\frac{2 \epsilon_{\perp}}{\epsilon_{\|}} f=0
$$

We suppose that in the annulus $b \leq r \leq a, \epsilon_{i}=\epsilon_{i, 0}\left(\frac{r}{a}\right)^{m}$, assuming as above subscripts $i=\|, \perp$. In this region, we then have

$$
r^{2} \frac{\partial^{2} f}{\partial r^{2}}+(m+2) r \frac{\partial f}{\partial r}-2 \frac{\epsilon_{\perp}}{\epsilon_{\|}} f=0,
$$

which now has an explicit solution

$$
f(r)=c_{1} r^{t_{1}}+c_{2} r^{t_{2}}
$$

with

$$
t_{1,2}=\frac{1}{2}\left(-(m+1) \pm \sqrt{(m+1)^{2}+8 \frac{\epsilon_{\perp, 0}}{\epsilon_{\|, 0}}}\right) .
$$

Inside $(r<b)$ and outside $(r>a)$ the annulus, the equations take the standard form

$$
r^{2} \frac{\partial^{2} f}{\partial r^{2}}+2 r \frac{\partial f}{\partial r}-2 f=0 .
$$

We can now write explicit forms for the potential in the three regions:

$$
\begin{aligned}
& \Phi(r, \theta)=E_{0} A r \cos \theta, \quad 0 \leq r \leq b, \\
& \Phi(r, \theta)=E_{0}\left(B r^{t_{1}}+C r^{t_{2}}\right) \cos \theta, \quad b \leq r \leq a, \\
& \Phi(r, \theta)=E_{0}\left(\frac{\tilde{\alpha}}{3} \frac{a^{3}}{r^{2}}-r\right) \cos \theta, \quad r \geq a,
\end{aligned}
$$


where we have factored out the external field $E_{0}$ which multiplies all coefficients. The quantity $\tilde{\alpha}$ is a polarizability for the inclusion, and is related to the effective inclusion dielectric $\tilde{\epsilon}$ by the usual Clausius-Mossotti relation:

$$
\frac{\tilde{\alpha}}{3}=\frac{\tilde{\epsilon}-1}{\tilde{\epsilon}+2} \Leftrightarrow \tilde{\epsilon}=\frac{3+2 \tilde{\alpha}}{3-\tilde{\alpha}}
$$

The Maxwell equations require boundary conditions of continuous $D_{r}$ and $E_{\theta}$ at $r=b$ and $r=a$. These yield

$$
\begin{aligned}
A b & =B b^{t_{1}}+C b^{t_{2}}, \\
\epsilon_{c} A & =\epsilon_{\|, 0} b^{m-1}\left(B t_{1} b^{t_{1}}+C t_{2} b^{t_{2}}\right), \\
B a^{t_{1}}+C a^{t^{2}} & =\left(\frac{\tilde{\alpha}}{3}-1\right), \\
\epsilon_{\|, 0} a^{m-1}\left(B t_{1} a^{t_{1}}+C t_{2} a^{t_{2}}\right) & =-\epsilon_{m}\left(\frac{2 \tilde{\alpha}}{3}+1\right),
\end{aligned}
$$

where we recall that $\epsilon_{c}$ is the dielectric function inside the core $(0 \leq r \leq b$. Some algebra then yields the following formula for the effective inclusion dielectric coefficient:

$$
\tilde{\epsilon}=\epsilon_{\|, 0} \frac{t_{2}\left[t_{1} \frac{\epsilon_{\|, 0}}{\epsilon_{c}}\left(\frac{b}{a}\right)^{m}-1\right]\left(\frac{b}{a}\right)^{\xi}-t_{1}\left[t_{2} \frac{\epsilon_{\|, 0}}{\epsilon_{c}}\left(\frac{b}{a}\right)^{m}-1\right]}{\left[t_{1} \frac{\epsilon_{\|, 0}}{\epsilon_{c}}\left(\frac{b}{a}\right)^{m}-1\right]\left(\frac{b}{a}\right)^{\xi}-\left[t_{2} \frac{\epsilon_{\|, 0}}{\epsilon_{c}}\left(\frac{b}{a}\right)^{m}-1\right]},
$$

where the auxiliary coefficient $\xi$ is given by

$$
\xi=\left[(m+1)^{2}+8 \frac{\epsilon_{\perp, 0}}{\epsilon_{\|, 0}}\right]^{1 / 2} .
$$

An analogous procedure can be followed if the core, rather than the shell, is radially anisotropic.

\section{Appendix B. Electrostatic formulas for cylindrical inclusions}

This calculation proceeds by close analogy with that in appendix A. The relevant cylindrical coordinates are now $\rho$, $\theta, z$, where we use $\theta$ as the azimuthal coordinate to avoid confusion with the electric potential $\Phi$. The shell is in the region $b \leq \rho a$. The components $\epsilon_{\|}$and $\epsilon_{\perp}$ now correspond to the $\mathbf{e}_{\rho}$ and $\mathbf{e}_{\theta}$ directions respectively. We suppose the electric field to be perpendicular to the axis of the cylinder. The coordinate $z$ is ignorable because of the translational symmetry with respect to $z$. The electric displacement is

$$
\mathbf{D}=\epsilon_{\|} \frac{\partial \Phi}{\partial \rho} \mathbf{e}_{\rho}+\epsilon_{\perp} \frac{1}{\rho} \frac{\partial \Phi}{\partial \theta} \mathbf{e}_{\theta}
$$

The condition $\nabla \cdot \mathbf{D}=0$ becomes

$$
\nabla \cdot \mathbf{D}=\frac{1}{\rho} \frac{\partial}{\partial \rho}\left(\rho \epsilon_{\|}(\rho) \frac{\partial \Phi}{\partial \rho}\right)+\epsilon_{\perp} \frac{1}{\rho^{2}} \frac{\partial^{2} \Phi}{\partial \theta^{2}}=0
$$

By analogy with eq. (A.3), the correct solution is given by the ansatz $\Phi(\rho, \theta)=f(\rho) \cos \theta$, and by analogy with eq. (A.5), this yields

$$
\nabla \cdot \mathbf{D}=\cos \theta\left[\frac{1}{\rho} \frac{\partial}{\partial \rho}\left(\rho \epsilon_{\|}(\rho) \frac{\partial f}{\partial \rho}\right)-\frac{1}{\rho^{2}} \epsilon_{\|}(\rho) f(\rho)\right]=0 .
$$

We now suppose that the local behavior of $\epsilon_{i}=\epsilon_{i, 0}\left(\frac{\rho}{a}\right)^{m}$. This leads to a general equation,

$$
\rho^{2} \frac{\partial^{2} f(\rho)}{\partial \rho^{2}}+(m+1) \rho \frac{\partial f(\rho)}{\partial \rho}-\frac{\epsilon_{\perp, 0}}{\epsilon_{\|, 0}} f(\rho)=0
$$

which applies explicitly in the annulus $b \leq \rho \leq a$, whereas inside the core $\rho \leq b$ and in the host medium $\rho \geq a$, where the dielectric is constant $(m=0)$ and isotropic, eq. (B.4) reduces to

$$
\rho^{2} \frac{\partial^{2} f(\rho)}{\partial \rho^{2}}+\rho \frac{\partial f(\rho)}{\partial \rho}-f(\rho)=0 .
$$


The analog of eq. (A.10) for the potential in the three regions is now

$$
\begin{aligned}
& \Phi(\rho, \theta)=E_{0} A \rho \cos \theta, \quad 0 \leq \rho \leq b, \\
& \Phi(\rho, \theta)=E_{0}\left(B \rho^{s_{1}}+C \rho^{s_{2}}\right) \cos \theta, \quad b \leq \rho \leq a, \\
& \Phi(\rho, \theta)=E_{0}\left(\frac{\tilde{\alpha}}{2} \frac{a^{2}}{\rho}-\rho\right) \cos \theta, \quad \rho \geq a,
\end{aligned}
$$

where the meaning of the parameters as follows:

a)

$$
s_{1,2}=\frac{1}{2}\left(-m \pm \sqrt{m^{2}+4 \frac{\epsilon_{\perp, 0}}{\epsilon_{\|, 0}}}\right)
$$

which is the cylindrical symmetry analogy of eq. (A.8).

b) $\tilde{\alpha}$ is a polarizability for the circular slice, now related to the effective inclusion dielectric $\tilde{\epsilon}$ by a two-dimensional version of the usual Clausius-Mossotti relation [59]:

$$
\frac{\tilde{\alpha}}{2}=\frac{\tilde{\epsilon}-1}{\tilde{\epsilon}+1} \Leftrightarrow \tilde{\epsilon}=\frac{2+\tilde{\alpha}}{2-\tilde{\alpha}}
$$

The final result for $\tilde{\epsilon}$ is essentially identical to the spherical analogue (eq. (A.12)), but with the quantities in the formula taking different values:

$$
\tilde{\epsilon}=\epsilon_{\|, 0} \frac{s_{2}\left[s_{1} \frac{\epsilon_{\|, 0}}{\epsilon_{c}}\left(\frac{b}{a}\right)^{m}-1\right]\left(\frac{b}{a}\right)^{\psi}-s_{1}\left[s_{2} \frac{\epsilon_{\|, 0}}{\epsilon_{c}}\left(\frac{b}{a}\right)^{m}-1\right]}{\left[s_{1} \frac{\epsilon_{\|, 0}}{\epsilon_{c}}\left(\frac{b}{a}\right)^{m}-1\right]\left(\frac{b}{a}\right)^{\psi}-\left[s_{2} \frac{\epsilon_{\|, 0}}{\epsilon_{c}}\left(\frac{b}{a}\right)^{m}-1\right]},
$$

with the auxiliary parameter $\psi$ given by (see eq. (A.12) for the spherical analogous spherical quantity)

$$
\psi=\left[m^{2}+4 \frac{\epsilon_{\perp, 0}}{\epsilon_{\|, 0}}\right]^{1 / 2} .
$$

\section{Appendix C. Particle internal homogenization versus exact solution of the Maxwell equations}

In sect. 4 we have used the dielectric mixing formulas of eqs. (6) and (7) for isotropic particles embedded in an anisotropic host. The formulas require as input the effective dielectric constant of the core-shell complex and the anisotropic dielectric components of the host medium. However, the calculation of the effective inclusion dielectric constant (see appendix B) has been carried out supposing the host medium to be isotropic, with the mean value of the diagonals of the dielectric tensor. In this case the electrostatics reduces to a standard problem and the calculation can be carried out in a straightforward manner. This procedure is open to question, and clearly would fail if the host dielectric constant were sufficiently anisotropic. It is therefore useful to approach this problem from another direction in order to test this approximation.

Thus here we use COMSOL Multiphysics ${ }^{\circledR}$ software to solve Maxwell's equations exactly for a core-shell system with an isotropic core, a radially anistropic shell, and an anisotropic host for particular values of parameters. The size of the cubic box has been chosen so as to include precisely one core-shell particle, so that the particle volume fractions in the whole system match in the "exact" calculation and in the approximate solution following the protocol of sect. 4. Although we have called this approach into question in sect. 1 for a number of reasons, good agreement between two different approaches, each using a different set of approximations, could be thought of as good evidence for the basic viability of either.

In fig. 17 we compare results of a calculation for the parallel component of the medium dielectric tensor using three methods. These are: i) COMSOL Multiphysics ${ }^{\circledR}$ modeling (blue in our online version); ii) our effective medium theory (red), and iii) an intermediate theory in which the real radially anisotropic shell is replaced by an isotropic shell whose dielectric constant is determined by the trace of the shell dielectric tensor (green). We present results for both the real and imaginary parts of the medium dielectric tensor. 

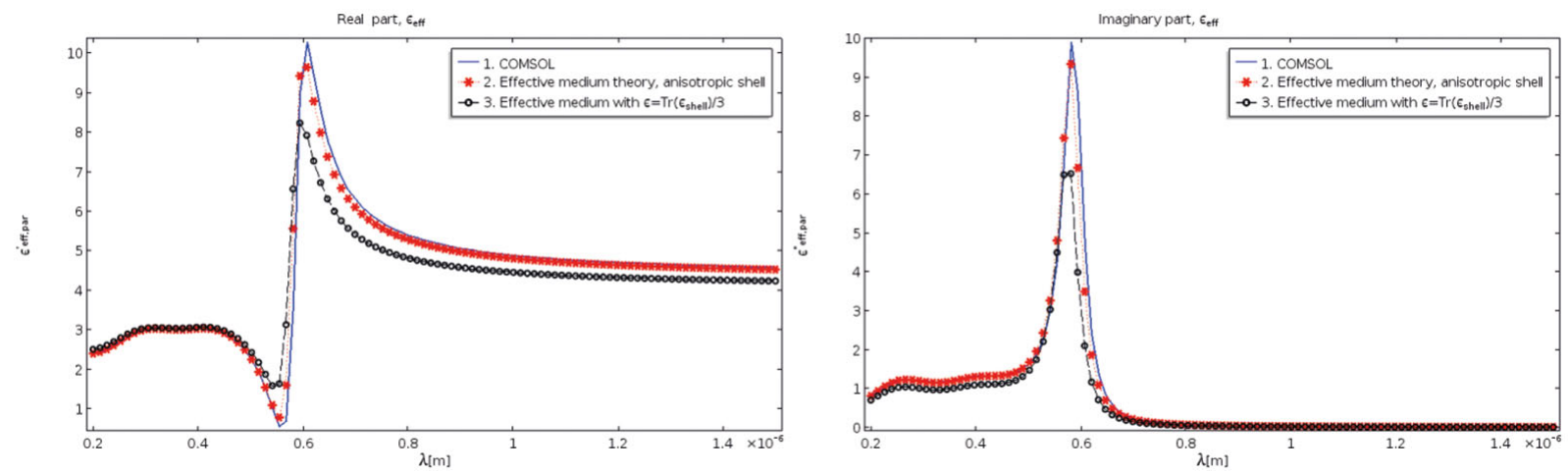

Fig. 17. Comparison of COMSOL Multiphysics ${ }^{\circledR}$ with effective medium solutions, described in sects. 3, 4 and appendix B. Parameters: Particle volume fraction $\eta=0.3$. Au core: $R_{\text {core }}=5 \mathrm{~nm}$; anisotropic dielectric tensor of shell $\epsilon_{r \text { shell }}=4 ; \epsilon_{t}$ shell $=2.5$; $f=0.4$. Host: $\epsilon_{\|}=1.7^{2}, \epsilon_{\perp}=1.5^{2}$, corresponding to the optical regime of a typical nematic liquid crystal. Plots (a), (b), respectively, real and imaginary parts of parallel components of effective medium dielectric tensor. For meanings of each curve, see text and in-figure legend.

The agreement between the curves predicted by our theory and the numerical results is remarkably good. The intermediate theory, which averages over the radial anisotropy in the shell departs significantly, both for the real and imaginary parts, from both the effective medium and the COMSOL Multiphysics ${ }^{\circledR}$ results. This departure is noticeable both in the position and the magnitude of the plasmon resonance-induced peaks. The effective medium and the COMSOL Multiphysics ${ }^{\circledR}$ results are however very close indeed.

However, there is a significant difference in the computational effort required to reach these results. Because all formulas are analytical in our effective medium theory, the effective medium curves in fig. 17 can be derived almost instantly on a laptop. On the other hand, the equivalent numerical curves require of the order of a day, and even then significant interpolation is required to derive a smooth curve as a function of frequency. If subsequent runs are required for new physical parameters, the effective medium method is considerably more versatile.

Open Access This is an open access article distributed under the terms of the Creative Commons Attribution License (http://creativecommons.org/licenses/by/4.0), which permits unrestricted use, distribution, and reproduction in any medium, provided the original work is properly cited.

\section{References}

1. A. Sihvola, Electromagnetic mixing formulas and applications (The Institution of Engineering and Technology, London, UK, 1999).

2. T.C. Choy, Effective medium theory: principles and applications (Clarendon Press, Oxford, UK, 1999).

3. N. Engheta, R.W. Ziolokowski (Editors), Metamaterials: Physics and Engineering Explorations (IEEE Press, Piscataway, N.J., 2006).

4. L. Solymar, E. Shamonina, Waves in Metamaterials (Oxford University Press, Oxford, UK, 2009).

5. W. Cai, V. Shalaev, Optical Metamaterials: Fundamentals and Applications (Springer, New York, 2010).

6. M.A. Noginov, G. Zhu et al., Nature 460, 1110 (2009).

7. D. Rodríguez-Fernández, J. Pérez-Juste, I. Pastoriza-Santos, L.M. Liz-Marzán, Chem. Open 1, 90 (2012).

8. I.C. Khoo, D.H. Werner, X. Liang, A. Diaz, B. Weiner, Opt. Lett. 31, 2592 (2006).

9. D. Rodríguez-Fernández, L.M. Liz-Marzán, Part. Part. Syst. Charact. 30, 46 (2013).

10. A. Karvounis, B. Gholipour, K.F. MacDonald, N.I. Zheludev, Appl. Phys. Lett. 109, 051103 (2016).

11. Z. Chen, Y. Gao, L. Kang, C. Cao, S. Chen, H. Luo, J. Mater. Chem. A 2, 2718 (2014).

12. A. Erbe, R. Sigel, Eur. Phys. J. E 22, 303 (2007).

13. P.A.A. De Beule, J. Opt. Soc. Am. A 31, 162 (2014).

14. A.B. Golovin, O.D. Lavrentovich, Appl. Phys. Lett. 95, 254104 (2009).

15. M. Warner, E.M. Terentjev, Liquid Crystal Elastomers (Oxford University Press, Oxford, UK, 2007).

16. L. Ferrari, C. Wu, D. Lepage, X. Zhang, Z. Liu, Progr. Quantum Electron. 40, 1 (2015).

17. V.P. Drachev, V.A. Podolskiy, A.V. Kildishev, Opt. Express 21, 15048 (2013).

18. W.R. Tinga, W. Voss, D. Blossey, J. Appl. Phys. 44, 3897 (1973) Table 1 of this paper includes a useful summary of mixture formulae with different ranges of applicability.

19. S.M. Shelestiuk, V.Y. Reshetnyak, T.J. Sluckin, Phys. Rev. E 83, 041705 (2011).

20. A. Sihvola, Electromagnetics 17, 269 (1997). 
21. A. Sihvola, Progr. Electromagn. Rese. 51, 65 (2005).

22. A. Lakhtakia, B. Michel, W.S. Weiglhofer, J. Phys. D 30, 230 (1997).

23. T.G. Mackay, A. Lakhtakia, Microwave Opt. Technol. Lett. 47, 313 (2005).

24. T.G. Mackay, A. Lakhtakia, J. Nanophoton. 6, 069501 (2012).

25. C.F. Bohren, D.R. Huffman, Absorption and Scattering of Light by Small Particles (John Wiley, New York, 1983).

26. J.C.M. Garnett, Philos. Trans. R. Soc. A 203, 385 (1904).

27. D.A.G. Bruggeman, Ann. Phys. (Leipzig) 416, 636 (1935).

28. R. Landauer, Electrical conductivity in inhomogeneous media, in Proceedings of the 1st Conference on Electrical Transport and Optical Properties of Inhomogeneous Media, Ohio State University, edited by J.C. Garland, D.B. Tanner (AIP, New York, 1977) pp. 2-45.

29. A. Ishimaru, Wave propagation and scattering in random media (Academic Press, New York, 1978).

30. D.J. Bergman, D. Stroud, Sol. State Phys. 46, 147 (1992).

31. D.J. Bergman, Phys. Rep. 43, 377 (1978).

32. T.G. Mackay, A. Lakhtakia, Modern analytical electromagnetic homogenization (Morgan \& Claypool Publishers, 2015).

33. V.A. Markel, J. Opt. Soc. Am. A 33, 1244 (2016).

34. V.A. Markel, J. Opt. Soc. Am. A 33, 2237 (2016).

35. V. Myroshnychenko, J. Rodríguez-Fernández, I. Pastoriza-Santos, A.M. Funston, C. Novo, P. Mulvaney, L.M. Liz-Marzán, F.J.G. de Abajo, Chem. Soc. Rev. 37, 1792 (2008).

36. B. Rodríguez-Gonzalez, F. Attouchi, M.F. Cardinal, V. Myroshnychenko, O. Stephan, F.J. Garcia de Abajo, L.M. LizMarzan, M. Kociak, Langmuir 28, 9063 (2012).

37. M.E. Mezeme, S. Lasquellec, C. Brosseau, Phys. Rev. E 81, 057602 (2010).

38. K.K. Karkkainen, A.H. Sihvola, K.I. Nikoskinen, IEEE Trans. Geosci. Remote Sens. 38, 1303 (2000).

39. S.C. Doanh, N.A. Bang, Physica B (2017) https://doi.org/10.1016/j.physb.2017.11.048.

40. H. Chen, C.T. Chan, P. Sheng, Nat. Mater. 9, 387 (2010).

41. S.A. Cummer, B. Popa, D. Schurig, D.R. Smith, J. Pendry, M. Rahm, A. Starr, Phys. Rev. Lett. 100, 024301 (2008).

42. S. Zhang, C. Xia, N. Fang, Phys. Rev. Lett. 106, 024301 (2011).

43. S. Guenneau, C. Amra, D. Veynante, Opt. Express 20, 8207 (2012).

44. I. Abdulhalim, M. Zourob, A. Lakhtakia, Electromagnetics 28, 214 (2008).

45. I. Abdulhalim, A. Lakhtakia, A. Lahav, F. Zhang, J. Xu, Proc. SPIE 7041, 70410C (2008).

46. E.D. Palik, Handbook of optical constants of solids (Academic Press, New York, 1998).

47. P.B. Johnson, R.W. Christy, Phys. Rev. B 6, 4370 (1972).

48. P.G. Etchegoin, E.C.L. Ru, M. Meyer, J. Chem. Phys. 125, 164705 (2006).

49. E. Coronado, G. Schatz, J. Chem. Phys. 119, 3296 (2003).

50. W.A. Kraus, G.C. Schatz, J. Chem. Phys. 79, 6130 (1983).

51. A. Moroz, J. Phys. Chem. C 112, 10641 (2008).

52. V.Y. Reshetnyak, I.P. Pinkevych, T.J. Sluckin, D.R. Evans, Opt. Express 24, A21 (2016).

53. S.A. Cummer, B.I. Popa, D. Schurig, D.R. Smith, J. Pendry, Phys. Rev. E 74, 036621 (2006).

54. W. Cai, U. Chettar, A. Kildishev, V. Shalaev, Nat. Photon. 1, 224 (2007).

55. A.A. Lucas, L. Henrard, P. Lambin, Phys. Rev. B 49, 2888 (1994).

56. V.Y. Reshetnyak, T.J. Sluckin, S.J. Cox, J. Phys. D 30, 3253 (1997).

57. S.J. Cox, V.Y. Reshetnyak, T.J. Sluckin, J. Phys. D 31, 1611 (1998).

58. A.D. Kiselev, V.Y. Reshetnyak, T.J. Sluckin, Phys. Rev. E 65, 056609 (2002).

59. H. Kettunen, H. Wallén, A. Sihvola, J. Appl. Phys. 114, 044110 (2013).

60. U.K. Chettiar, N. Engheta, Opt. Express 20, 22976 (2012).

61. X. Yu, L. Gao, Phys. Lett. A 359, 516 (2006).

62. K.-H. Kim, Y.-S. No, S. Chang, J.-H. Choi, H.-G. Park, Sci. Rep. 5, 16027 (2015).

63. H.N.S. Krishnamoorthy, Y. Zhou, S. Ramanathan, E. Narimanov, V.M. Menon, Appl. Phys. Lett. 104, 121101 (2014).

64. C.Y.C. Wu, M. Chu, Int. J. Nanomed. 6, 807 (2011).

65. B.J. Jankiewicz, D. Jamiola, J. Choma, M. Jaroniec, Adv. Colloids Interface Sci. 170, 28 (2012).

66. N. Elbialy, N. Mohamed, A.S. Monem, Micropor. Mesopor. Mater. 190, 197 (2014).

67. C. Mätzler, MATLAB Functions for Mie Scattering and Absorption, Research Report No. 2002-8 (Oxford, UK, 2002).

68. J.Y. Lu, A. Raza, N.X. Fang, G. Chen, T. Zhang, J. Appl. Phys. 120, 163103 (2016). 